\title{
Disc-like Objects in Hierarchical Hydrodynamical Simulations: Comparison with Observations
}

\author{
A. Sáiz, ${ }^{1}$ R. Domínguez-Tenreiro, ${ }^{1}$ P.B. Tissera ${ }^{2}$ and S. Courteau ${ }^{3}$ \\ ${ }^{1}$ Departamento de Física Teórica, C-XI. Universidad Autónoma de Madrid, Madrid, E-28049, Spain \\ ${ }^{2}$ I.A.F.E., Casilla de Correos 67, Suc. 28, Buenos Aires, 1428, Argentina \\ ${ }^{3}$ University of British Columbia, Dept. of Physics and Astronomy, Vancouver, BC, Canada V6T $1 Z 1$
}

1 February 2008

\begin{abstract}
We present results from a careful and detailed analysis of the structural and dynamical properties of a sample of 29 disc-like objects identified at $z=0$ in three AP3M-SPH fully consistent cosmological simulations. These simulations are realizations of a CDM hierarchical model, where an inefficient Schmidt law-like algorithm to model the stellar formation process has been implemented. We focus on properties that can be constrained with available data from observations of spiral galaxies, namely, the bulge and disc structural parameters and the rotation curves. Comparison with data from Broeils (1992), de Jong (1996) and Courteau $(1996,1997)$ gives satisfactory agreement, in contrast with previous findings using other codes. This suggests that the stellar formation implementation we have used has succeeded in forming compact bulges that stabilize disc-like structures in the violent phases of their assembly, while in the quiescent phases the gas has cooled and collapsed according with the Fall \& Efstathiou standard model of disc formation.
\end{abstract}

Key words: galaxies: evolution - galaxies: formation - galaxies: structure galaxies: spiral - hydrodynamics - methods: numerical - cosmology: theory dark matter

\section{INTRODUCTION}

Understanding how galaxies form and evolve into the objects we observe today remains one of the most fundamental quest of astrophysical research. Even if the field is still at its beginnings, the use of numerical approaches to study how galaxies are assembled in a cosmological hierarchical scenario from primordial fluctuations, seems promising. The main advantage of these approaches (namely hydrodynamical simulations) is that physics is introduced at a most general level, and the dynamical processes relevant to galaxy assembly (i.e., collapse, gas infall, interactions, mergers, instabilities...) emerge naturally, rather than by assumption, and can be followed in detail. Only the star forming processes need to be modelled. These considerations emphasize the interest of hydrodynamical simulations as an outstanding working tool to learn about galaxy formation and evolution from primordial fluctuations. For other approaches to this problem, see Manrique et al. (in preparation), Firmani \& Avila-Reese (2000) and the references therein.

As discs are naturally produced in the collapse of a dissipative system embedded in a gravitational field, learning how to get well-behaved discs (i.e., similar to those observed in spirals) in such simulations is one important step towards understanding galaxy assembly in general. According to the standard model of disc formation (Fall \& Efstathiou 1980), 
disc-like structures with observable counterparts can form provided that the diffuse gas in the dark matter halo cools and collapses conserving its specific angular momentum, $\boldsymbol{j}$. In hierarchical scenarios, halo gas cooling occurs with $\boldsymbol{j}$ conservation in the quiescent phases of galaxy assembly, producing extended, thin discs with an exponential mass density profile in the equatorial plane (Dalcanton, Spergel \& Summers 1997). But violent phases play also a fundamental role in these scenarios. During these phases, the system undergoes interactions and merger events, that can destroy discs that had possibly formed in previous quiescent phases.

Thus far, no hydrodynamical simulation of galaxy formation in fully consistent hierarchical cosmological scenarios had provided extended discs, with structural and dynamical properties similar to those observed in spiral galaxies (see, however, recent work by SommerLarsen \& Dolgov 1999). The objects formed were either a) gaseous and too concentrated, when no stellar formation processes are considered at all (Navarro \& Benz 1991; Navarro, Frenk \& White 1995; Evrard, Summers \& Davis 1994; Vedel, Hellsten \& Sommer-Larsen 1994; Navarro \& Steinmetz 1997; Sommer-Larsen, Gelato \& Vedel 1999; Weil, Eke \& Efstathiou 1998 and references therein), or b) rather stellar and without discs, when these processes have been included (Navarro \& Steinmetz 2000; Thacker \& Couchman 2000). In the former cases, objects are too concentrated due to an excessive $\boldsymbol{j}$ loss in violent events; this is the so-called disc angular momentum catastrophe [DAMC]. In the latter cases, as discussed by Tissera, Sáiz \& Domínguez-Tenreiro (in preparation, hereafter TSDT) the particular star formation implementations used lead to a too early gas transformation into stars, leaving no gas to cool and form discs at lower $z$. The effects of star formation have also been considered by Katz (1992) and Steinmetz \& Müller (1995) who obtained, in both cases, a three component system that resembles a spiral galaxy. However, their simulations treat only the case of a semi-cosmological modellization of the collapse of a constant density perturbation in solid-body rotation. Sommer-Larsen \& Dolgov (1999) have succeeded in avoiding excessive $\boldsymbol{j}$ loss in warm dark matter scenarios. Nevertheless, their discs still do not match observations very well.

This inability to reproduce observed discs in fully consistent cosmological simulations, represents clearly a major pitfall of present-day numerical simulations as a working tool to learn about galaxy formation and evolution. In a series of papers (Domínguez-Tenreiro, Tissera \& Sáiz 1998; Sáiz, Tissera \& Domínguez-Tenreiro 1999; TSDT) it has been shown that the object properties at the end of violent phases of evolution critically depend on the structural stability of the disc-like objects involved in interaction and merger events, on the one hand, and on the availability of gas to regenerate a disc structure after the last violent episode, on the other hand. Concerning stability, cold thin discs are known to be very unstable against non-axisymmetric modes (Athanassoula \& Sellwood 1986; Binney \& Tremaine 1987; Barnes \& Hernquist 1991, 1992; Martinet 1995 and references therein; Mihos \& Hernquist 1994, 1996; Mo, Mao \& White 1998). Massive haloes can stabilize discs, but not every halo is able to stabilize the pure exponential disc that would form from $\boldsymbol{j}$-conserving diffuse gas collapse in its last quiescent phase. In these cases, a central compact bulge is needed to ensure stability (Christodoulou, Shlosman \& Tohline 1995; van den Bosch 1998, 2000). Otherwise, non-axisymmetric instabilities could be easily triggered by interactions and mergers, causing important $\boldsymbol{j}$ losses (that is, DAMCs), followed by strong gas inflow.

In that series of papers we also show that compact central bulges form when star-forming processes are considered in hierarchical hydrodynamical simulations, provided that the particular star formation implementation used does not lead to a too early gas exhaustion (i.e., inefficient star formation, see Silk 1999). The physical processes involved in disc formation

(C) 0000 RAS, MNRAS 000, 000-000 
and stability when an inefficient Schmidt law-like stellar formation algorithm is implemented in these simulations are discussed in detail in TSDT.

The aim of this paper is to show that discs identified in galaxy-like objects produced in these hierarchical hydrodynamical simulations are, at a structural and dynamical level, similar to those observed in spirals. To this end, we analyze the structural and dynamical properties of a sample of 29 disc-like objects formed in three different such simulations. We focus on those properties that can be constrained with available data for observed spiral galaxies. These data are taken principally from the compilations of galaxy structural parameters of Broeils (1992, hereafter B92), de Jong (1996) and Courteau (1996, 1997b, hereafter C97b).

The paper is organized as follows: $\$ 2$ describes the simulations. The general characteristics of disc-like objects at $z=0$ are outlined in $\S 3$, and their bulge-disc decomposition and rotation curves are discussed in $\S$ 团 and $\S 5$, respectively. Finally, a summary and conclusions are presented in $\$ 6$.

\section{THE SIMULATIONS}

We consider three simulations, S1, S1b, and S1c太, from different realizations of a given model for the primordial spectrum of the density fluctuation field. In each case, we follow the evolution of $64^{3}$ gas plus dark matter particles in a periodic box of comoving side $10 \mathrm{Mpc}$ $\left(H_{0}=100 h \mathrm{~km} \mathrm{~s}^{-1} \mathrm{Mpc}^{-1}\right.$ with $\left.h=0.5\right)$ using a SPH code coupled to a high resolution AP3M code (Thomas \& Couchman 1992; see Tissera, Lambas \& Abadi 1997 for details on SPH algorithm implementation). The initial distributions of positions and velocities of the $64^{3}$ particles are consistent with a standard CDM cosmology, with $\Omega_{\mathrm{b}}=0.1, \Lambda=0$ and $b=2.5$; one out of ten of them is randomly chosen to be a gas particle. These initial distributions are set by means of the ACTION algorithm, kindly provided by E. Bertschinger. All, dark, gas and stellar particles have the same mass, $2.6 \times 10^{8} \mathrm{M}_{\odot}$. AP3M-SPH integrations were carried out from $z=10$ to the present using fixed time steps $\Delta t=1.3 \times 10^{7}$ years. The gravitational softening length is $3 \mathrm{kpc}$ and the minimum allowed smoothing length is $1.5 \mathrm{kpc}$. These simulations include a star formation prescription described by Tissera et al. (1997; see Tissera 2000 for a discussion on the resulting star formation history). The gas cools due to radiative cooling. We use the approximation for the cooling function by Dalgarno \& McCray (1972) for a primordial mixture of hydrogen and helium. Gas particles are transformed into stars if they are cold $\left(T_{*}<3 \times 10^{4} \mathrm{~K}\right)$, dense $\left(\rho>\rho_{\text {crit }} \approx 7 \times 10^{-26}\right.$ $\mathrm{g} \mathrm{cm}^{-3}$ ), in a convergent flow and satisfy Jean's instability criterion. When a gas particle satisfies these conditions, it is transformed into a star particle after a time interval $(\tau)$ over which its gas mass is being converted into stars according to

$\frac{\mathrm{d} \rho_{\mathrm{gas}}}{\mathrm{d} t}=-\frac{\mathrm{d} \rho_{\text {stars }}}{\mathrm{d} t}=-c \frac{\rho_{\text {gas }}}{t_{*}}$,

where $c$ is the star formation efficiency, $t_{*}$ is a characteristic time-scale assumed to be proportional to the dynamical time of the particle $\left(t_{*} \propto \rho_{\text {gas }}^{-1 / 2}\right)$ and $\tau=\ln 100 t_{*} / c$ is the time interval over which 99 per cent of the gas mass in a particle is expected to be transformed into stars (Navarro \& White 1993). We adopt a low star formation [SF] efficiency with $c=0.01$ (for a discussion of the inefficient SF implementation, see TSDT). No supernovae explosion effects have been considered. However, this low $c$ value avoids the quick depletion of gas at

\footnotetext{
* Simulation S1 has been studied in Domínguez-Tenreiro et al. (1998) and Sáiz et al. (1999), and in Tissera \& DomínguezTenreiro (1998) (their I.2 simulation).
} 
high $z$, leaving enough remanent gas to form disc-like structures at low $z$. To some extent, this could mimic the effects of energy injection from supernovae explosions. The treatment of supernova feedback in numerical simulations is still an open and complex problem that remains to be properly solved.

\section{SIMULATED OBJECTS AT $z=0$}

Galaxy-like objects are identified at their virial radius, $r_{200}$, at $z=0$. Dark matter haloes formed in the S1 simulation have been extensively studied in Tissera \& Domínguez-Tenreiro (1998, hereafter TDT98). These haloes, as well as those formed in S1b and S1c simulations, are resolved with a relatively high number of particles (see the number of dark mass particles, $N_{\text {dark }}$, per halo, in Table (1).

The main baryonic objects embedded in these dark haloes can be identified by applying a friends-of-friends (FOF) algorithm to the baryonic particle distribution, with a linking length of 10 per cent the mean total interparticle separation in the box. Baryonic objects identified in the simulation at $z=0$ are galaxy-like objects (GLOs) that span a range of morphological characteristics: disc-like objects (hereafter, DLOs), spheroids and irregular objects. DLOs have external extended, populated discs, consisting mostly of gas, central stellar bulge-like concentration and, in some cases, stars in a thick disc. Spheroids are mostly composed of stars forming a relaxed, spheroidal configuration, with a small amount of gas forming a small central disc. Irregular objects do not have a defined morphology, and in most cases they are the end product of a recent merger. In this paper only DLOs will be analyzed. Only those GLOs with a total baryon number $N_{\text {baryon }}=N_{\text {star }}+N_{\text {gas }}$ larger than $N_{\text {baryon }}^{\text {lim }}=150$ have been considered This criterion has been chosen as a compromise: taking $N_{\text {baryon }}^{\text {lim }}$ much higher than 150 would result into too few DLOs in our sample; taking $N_{\text {baryon }}^{\text {lim }}$ much lower than 150 would result into DLOs composed of too few particles to be properly analyzed.

Out of our 3 simulations, we are able to identify different numbers of discs $(9,13$ and 7, for a total sample of 29 objects) and spheroids or irregular objects (5, 7 and 11, not studied in this paper) following the previous mass-limit criterion. DLOs are labelled by the three coordinates (in $10 \times$ mesh units) that identify the cell where the centre of mass of their host halo is found in the total box. When several haloes (hosting either DLOs, spheroids or irregulars) are found in a given cell, a Greek letter is added to distinguish among them. Some haloes host more than one baryonic object; asterisks in Table 1 identify haloes that include more than one baryonic object (either DLO, spheroid or irregular). In this case, the different components are distinguished by means of a capital letter in order of decreasing mass. For instance, DLO \#165C (in S1b) belongs to a massive halo, where the more massive objects \#165A (spheroid) and \#165B (irregular) are also found; DLOs \#233A and \#233B

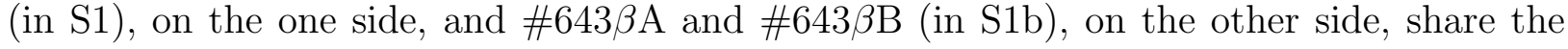
same halo.

The virial radius, $r_{200}$, maximum limiting radius, $R_{\max }$ (see $\S$ 田), dark, star and gas particle number, $N_{\text {dark }}, N_{\text {star }}$ and $N_{\text {gas }}$, respectively, within spheres of radii $r_{200}$ and $R_{\text {max }}$ for each of the 29 DLOs of the sample are listed in Table 1 .

In Fig. 1(a) we plot, for the $i$-th baryonic particle inside the halo hosting DLO \#242,

$\dagger$ For isolated GLOs, $N_{\text {baryon }}$ is defined unambiguously as $N_{\text {baryon }}=N_{\text {baryon }}^{\text {FoF }}$, where $N_{\text {baryon }}^{\text {FoF }}$ is the baryon number as given by the friends-of-friends algorithm for a given GLO. However, some GLOs have close satellites that are included in the friendsof-friends algorithm, even if these satellites are dynamically and structurally different entities from the GLO itself. In this case, the actual GLO baryon number is $N_{\text {baryon }}=N_{\text {baryon }}^{\text {FoF }}-\sum_{i} N_{\text {baryon }}^{\text {sat } i}$, with $N_{\text {baryon }}^{\text {sat, } i}$ the number of baryonic particles in the $i$-th satellite entering in $N_{\text {baryon }}^{\mathrm{FOF}}$.

(C) 0000 RAS, MNRAS 000, 000-000 
the cosine of the angle formed by its position and velocity vectors, $\boldsymbol{r}_{i}$ and $\boldsymbol{v}_{i}$, versus $r_{i}$, its distance to the DLO mass centre. In Fig. 1(b) we plot $\left|j_{\mathrm{z}, i}\right|$ versus $r_{i}$, where $j_{\mathrm{z}, i}$ is the component of the angular momentum per unit mass of the $i$-th particle parallel to $\boldsymbol{j}_{\text {dis }}$ (disc specific total angular momentum). The solid line is $V_{\text {cir }}(R) R$, where $V_{\text {cir }}(R)$ is the circular velocity at projected distance $R$ (see $\oint 5$ ). In these Figures, stars stand for stellar particles, circles for gas particles and open symbols for counter-rotating particles (i.e., with $j_{\mathrm{z}, i}<0$ ) of any kind. These Figures show that most gas particles placed at $r_{i} \lesssim 30 \mathrm{kpc}$ move coherently along circular trajectories (so that they have $\cos \left(\boldsymbol{r}_{i}, \boldsymbol{v}_{i}\right) \simeq 0$, see Fig. 1(a)) on the equatorial plane (and so they verify $\cos \left(\boldsymbol{j}_{i}, \boldsymbol{j}_{\text {dis }}\right) \simeq 1$ ), that is, they have $j_{\mathrm{z}, i} \simeq\left|\boldsymbol{j}_{i}\right| \simeq V_{\text {cir }}\left(R_{i}\right) R_{i}$, with a small dispersion around this value (see Fig. 1(b); $R_{i} \simeq r_{i}$ if the $i$-th particle is on the equatorial plane): the particles form a cold thin disc. In contrast with disc gas particles, gas particles placed at $r_{i} \gtrsim 30 \mathrm{kpc}$ (hereafter, halo gas particles) do not show any order: roughly half of them are in counter-rotation (open circles), their $\cos \left(\boldsymbol{r}_{i}, \boldsymbol{v}_{i}\right) \in[-1,1]$ and their $\left|j_{\mathrm{z}, i}\right|$ takes any value under the solid line.

Concerning stars, we see that most of them are found at $r_{i} \lesssim 2 \mathrm{kpc}$, where they form a central compact relaxed structure, i.e., a bulge, with $\boldsymbol{j}_{i}$ without any preferred direction and very low $\left|j_{z, i}\right|$; in some DLOs, as Figs. 1(a) and 1(b) illustrate, some stars are placed at $r_{i} \gtrsim 2 \mathrm{kpc}$ in some orderly fashion, forming a sort of thick disc. Baryons forming other DLOs in our sample follow similar behaviour patterns.

In Fig. 2 we plot the specific total angular momentum at $z=0$ versus mass for dark haloes, $j_{\mathrm{dh}}$ (open symbols), for the inner 83 per cent of the disc gas mass (i.e., the mass fraction enclosed by $R_{\mathrm{opt}} \equiv 3.2 R_{\mathrm{d}}$ in a purely exponential disc, where $R_{\mathrm{d}}$ is the disc scale length, see $\oint^{\text {A }}, j_{\mathrm{g}}$ (filled symbols), and for the stellar component of the DLOs in our simulations, $j_{\mathrm{s}}$ (starred symbols). We see that $j_{\mathrm{g}}$ is of the same order as $j_{\mathrm{dh}}$, so that these gas particles have collapsed conserving, on average, their angular momentum. Moreover, DLOs formed in our simulations are inside the box defined by observed spiral discs in this plot (Fall 1983). In contrast, $j_{\mathrm{s}}$ is much smaller than either $j_{\mathrm{dh}}$ or $j_{\mathrm{g}}$, meaning that the stellar component in the central parts has formed out of gas that had lost an important amount of its angular momentum.

A detailed description of the physical processes leading to DLO configuration at $z=0$ we have just described is given in Domínguez-Tenreiro et al. (1998) and TSDT. Here these processes are only briefly summarized:

The net effect of shocks and cooling in the quiescent phases of evolution on disordered halo gas particles, is that they force them to a coherent rotation with global specific angular momentum conservation. Consequently, in the quiescent phases of the evolution, gas particles tend to settle at the inner halo regions, and if the gravitational potential has some centre of axial (or central) symmetry at these regions, gas particles will move on a plane on circular orbits around this centre, forming a cold thin disc. Most stars in the central bulges have been formed out of gas particles that had been involved in an inflow event with high $\boldsymbol{j}$ loss; these compact central bulges create a gravitational potential that is spherically symmetric on scales of a few kpc, ensuring $\boldsymbol{j}$ conservation. This stabilizes the system against gas inflows in future violent phases of the evolution. In fact, in the next major merger event, those gas particles involved in the merger and with high $\boldsymbol{j}$ will be placed on an intermediate disc; in their turn, the stellar bulge of the smaller DLO involved in the event is eventually destroyed by strong tidal forces; then, incomplete orbital angular momentum loss puts most of its stars at some distance of the centre, where, after some relaxation, they form a kind of thick disc. Finally, most of the disc external gas particles are supplied by infall of particles, either belonging to baryonic clumps (satellites) or diffuse component, completing the DLO assembly. Because of the SF implementation used, gas in discs are not transformed into stars. Then, simulated 
discs are mostly composed of gas. Observed discs in spirals are mostly stellar, but the stellar discs inherit the structural and dynamical characteristics of the gaseous discs out of which disc stars have formed, so that, for the sake of comparison, the value of the parameters describing those properties can be safely determined from the simulated gaseous discs. A proper implementation of star formation with supernovae feedback should lead to a selfregulated star formation that would allow the formation of both compact stellar bulges and thin stellar discs.

\section{BULGE-DISC DECOMPOSITION}

Observationally, the structural parameters of spirals are obtained from a fit to the shape of the surface brightness profile. The galaxy luminosity profile is often decomposed into a bulge (b) and disc (d) component, such as

$\Sigma^{\text {light }}(R)=\Sigma_{\mathrm{b}}^{\text {light }}(R)+\Sigma_{\mathrm{d}}^{\text {light }}(R)$.

Each individual profile can be modelled using the parametrization from Sérsic (1968),

$\Sigma_{\mathrm{c}}^{\text {light }}(R)=\Sigma_{\mathrm{c}}^{\text {light }}(0) \exp \left[-\left(\frac{R}{R_{\mathrm{c}}}\right)^{1 / n}\right]$, for $\mathrm{c}=\mathrm{b}, \mathrm{d}$,

and where $n$ is a shape parameter taken to be $n=1$ for the disc component (i.e., a pure exponential law) and that is left as a free parameter for the bulge component. The de Vaucouleurs profile for spheroids corresponds to a choice of $n=4$. $\Sigma_{\mathrm{c}}^{\text {light }}(0)$ and $R_{\mathrm{c}}$ are the central surface brightness and scale length for the bulge and disc components.

The same profile form has been fit to the projected mass density of baryons, $\Sigma^{\text {mass }}(R)$, for the DLOs in our sample. $\Sigma^{\text {mass }}(R)$ is calculated by averaging concentric rings centred at the centre of mass of each DLO. Projections on the plane normal to the total angular momentum of each DLO (disc plane) have been used. These projected densities are binning dependent and somewhat noisy. To circumvent these problems, the integrated projected mass density in concentric cylinders of radius $R$ and mass $M^{\text {cyl }}(R)$,

$M^{\mathrm{cyl}}(R)=2 \pi \int_{0}^{R} \Sigma^{\mathrm{mass}}\left(R^{\prime}\right) R^{\prime} \mathrm{d} R^{\prime}$,

has been used as fitting function, instead of the projected mass density itself. Cumulative histograms $M^{\text {cyl }}\left(R_{i}\right)$ are constructed by adding equal height steps at those positions $R_{i}$ where baryon particles are placed. Fig. 3 shows these $M^{\mathrm{cyl}}\left(R_{i}\right)$ histograms for two DLOs. Simulated discs, as well as discs observed in spirals, do not have a sharp end. We have taken as maximum limiting radius for the simulated discs $R_{\max }=4.5 R_{\mathrm{d}}$ 尚 which corresponds to a limiting or truncation radius in surface brightness of $B=26.5 \mathrm{mag} \operatorname{arcsec}^{-2}$ assuming that discs have $B(0) \simeq 21.65 \mathrm{mag} \operatorname{arcsec}^{-2}$. As minimum limiting radius we have taken $R_{\min }=1.5 \mathrm{kpc}$. B . Because the total baryon number inside $R_{\max }$ for each object is known (see Table 1; this is equivalent to knowing the total DLO mass), we are left with four free fitting parameters among $n, \Sigma_{\mathrm{b}}(0), R_{\mathrm{b}}, \Sigma_{\mathrm{d}}(0)$ and $R_{\mathrm{d}}$. An updated version of the MINUIT software from CERN library has been used to make the fits.

$\ddagger$ A recent analysis by Pohlen, Dettmar \& Lütticke (2000) suggests a shallower truncation radius, with $R_{\max } \sim 3 R_{\mathrm{d}}$.

$\S$ The fitting determination of bulge/disc parameters is stable against changes in $R_{\text {min }}$. These changes affect mainly the $R_{\mathrm{b}, \text { eff }}$ parameter values, that in the less favoured case, $R_{\text {min }}=0.0 \mathrm{kpc}$, change on average only by less than a 10 per cent; $R_{\mathrm{d}}$ variations, on the other hand, are on average lower than the corresponding Monte Carlo errors due to finite sampling (see Fig. (1).

(C) 0000 RAS, MNRAS 000, 000-000 
To compare the size of bulges with different shape (i.e., with different bulge shape parameter $n$ ) the $R_{\mathrm{b}}$ parameter is meaningless because its value, as determined from the fitting procedure, depends on $n$. In fact, when bulges with the same physical scale and different shape are considered, $R_{\mathrm{b}}$ varies by orders of magnitude when $n$ varies by a factor of some few units (see Eqs. (5) and (6) below). To circumvent this shortcoming, an effective bulge scale length or half light radius, $R_{\mathrm{b}, \text { eff }}$, is often used by observers. In our case, it will be defined through the projected bulge mass profile by the condition $M_{\mathrm{b}}^{\text {cyl }}\left(R_{\mathrm{b} \text {,eff }}\right)=M_{\mathrm{b}} / 2$, where $M_{\mathrm{b}}$ is the bulge mass (i.e., it is a half mass radius). $R_{\mathrm{b}, \text { eff }}$ is related to $R_{\mathrm{b}}$ through:

$\gamma\left(2 n,\left(\frac{R_{\mathrm{b}, \mathrm{eff}}}{R_{\mathrm{b}}}\right)^{1 / n}\right)=\frac{\Gamma(2 n)}{2}$,

where $\gamma(m, u)$ and $\Gamma(m)$ are the incomplete and complete gamma functions. For $n=1$, Eq. (5) gives $\frac{R_{\mathrm{b}, \text { eff }}}{R_{\mathrm{b}}}=1.678$; for $n=4$ one finds $\frac{R_{\mathrm{b}, \mathrm{eff}}}{R_{\mathrm{b}}}=(7.669)^{4}$. For arbitrary $n$, Eq. (5) can be approximated by

$\frac{R_{\mathrm{b}, \mathrm{eff}}}{R_{\mathrm{b}}} \simeq(2.0 n-0.324)^{n}$.

The results for the effective bulge scale length, $R_{\mathrm{b}, \text { eff }}$, and disc scale length, $R_{\mathrm{d}}$, corresponding to the optimal bulge shape parameter, $n_{\mathrm{opt}}$, and, also, to $n=1$ (exponential bulge profile) are given in Table 2, together with their corresponding $\chi^{2}$ per degree of freedom parameter. For comparison, Table 2 also gives the disc scale length and the $\chi^{2}$ per degree of freedom for a de Vaucouleurs profile $(n=4)$. Note that the disc scale parameter, $R_{\mathrm{d}}$, is, in most cases, nearly independent of the bulge shape parameter (either $n_{\text {opt }}, n=1$ or $n=4$ ). Knowing the length and mass scale parameters for simulated bulges and discs allows us to determine their mass bulge-to-disc ratios,

$\frac{B}{D}=\frac{\Gamma(2 n+1)}{2}\left(\frac{R_{\mathrm{b}}}{R_{\mathrm{d}}}\right)^{2} \frac{\sum_{\mathrm{b}}^{\operatorname{mass}}(0)}{\sum_{\mathrm{d}}^{\operatorname{mass}}(0)}$.

These ratios are given in Table 2. This Table shows another interesting parameter characterizing DLO structure: the transition radius, $R_{\text {trans }}$, where $\Sigma_{\mathrm{b}}^{\text {mass }}(R)=\Sigma_{\mathrm{d}}^{\text {mass }}(R)$.

In Fig. 3 we have drawn the best fits to the integrated projected baryon mass density, $M^{\text {cyl }}(R)$, for DLOs \#242 and \#643 $\beta \mathrm{A}$. A solid line arrow marks $R_{\text {trans }}$; a dashed line arrow marks the lower limit of the fitting interval $R_{\min }=1.5 \mathrm{kpc}$. This Figure illustrates the good quality achieved in the fittings, even for the regions where $R<R_{\min }$, and hence the stability of the bulge/disc parameter determination against changes in $R_{\min }$, as mentioned before.

We see in Table 2 that bulges for most DLOs are more adequately modelled with an exponential profile $(n=1)$ than with a de Vaucouleurs profile $(n=4)$. This agrees with observational results for early and late-type spiral galaxies (e.g. Andredakis, Peletier \& Balcells 1995; Courteau, de Jong \& Broeils 1996 [CdJB96]; Moriondo, Giovanelli \& Haynes 1999 [MGH99]). Furthermore, Andredakis et al. (1995) and CdJB96 have found that the bulge shape parameter is correlated with spiral type. In particular, CdJB96 find that most $\mathrm{Sb}-\mathrm{Sc}$ and all high surface brightness spiral galaxies of later type are best fitted with a $n=1$ profile, most Sa-Sab galaxies are best modelled with a $n=2$ bulge, and only a small fraction of late type spirals have bulges that follow the de Vaucouleurs law. This distribution of shape parameters, $n_{\mathrm{opt}}$, is most similar to that of DLOs in Table 2. Based on the grid of shape parameters in Andredakis et al. (1995) and CdJB96, most of our simulated DLOs seem to be of type $T \geq 1$ or greater (see $n_{\text {opt }}$ in Table 21). The dependence of the observed $n$ values on the morphological type suggests a difference in the formation and evolution of the bulge component. 
The values of the $R_{\mathrm{b}}$ and $R_{\mathrm{d}}$ parameters, corresponding to a double exponential fit, are plotted in Fig. 4 for all the DLOs in our sample; squares, triangles and circles correspond to DLOs formed in S1, S1b and S1c simulations, respectively. The error bars in the top left corner of the Figure are the typical dispersions in the $R_{\mathrm{b}}$ (vertical) and $R_{\mathrm{d}}$ (horizontal) parameters values, arising from finite sampling and calculated through Monte Carlo realizations of the DLO profiles, as given by Eqs. (2) and (3), with $N_{\text {baryon }}$ particles and $n=1$. For comparison, in Fig. W we also show the $R_{\mathrm{b}}$ and $R_{\mathrm{d}}$ values given by CdJB96 and Courteau (1997a) from one-dimensional luminosity decompositions of Sb-Sc spirals (points). We get average bulge/disc scale length ratios of $R_{\mathrm{b}} / R_{\mathrm{d}}=0.09 \pm 0.03$ for DLOs in $\mathrm{S} 1, R_{\mathrm{b}} / R_{\mathrm{d}}=0.07 \pm 0.03$ for DLOs in S1b and $R_{\mathrm{b}} / R_{\mathrm{d}}=0.12 \pm 0.03$ for DLOs in S1c. The ratio for the whole sample of 29 DLOs is $R_{\mathrm{b}} / R_{\mathrm{d}}=0.09 \pm 0.04$, which compares well with the values found by CdJB96 in the $r$-band, $0.13 \pm 0.07$, and de Jong (1996) at $K$ alone, $0.09 \pm 0.04$. MGH99 also find $R_{\mathrm{b}} / R_{\mathrm{d}}=0.11 \pm 0.06$. In all cases, consistency between available data and our DLO sample is good. Note that we miss DLOs with small values of $R_{\mathrm{b}}$ and/or $R_{\mathrm{d}}$ as compared with the range of values quoted by these authors. It simply reflects our lack of dynamical resolution to resolve smaller structures.

In Fig. \# we compare scale lengths obtained from one-dimensional surface brightness decompositions with scales obtained from projected mass density decompositions with bulge shape parameter $n=1$. As compared with the disc, the bulge is typically less prominent in surface brightness than in projected mass density, since its mass-to-light ratio $\left(\Upsilon_{\mathrm{b}}\right)$ is likely to be higher than the disc mass-to-light ratio $\left(\Upsilon_{\mathrm{d}}\right)$. It is not clear, a priori, if this fact makes the previous comparisons meaningless. To find out if this is indeed the case, one would have to compare the values of the $R_{\mathrm{b}}$ and $R_{\mathrm{d}}$ parameters as obtained from a fit to the surface brightness profile of a given DLO with those obtained from a fit to its projected mass density profile. Since this is not possible, in order to assess the feasibility of the previous comparisons, we adopt the following method:

Assuming that both $\Upsilon_{\mathrm{b}}$ and $\Upsilon_{\mathrm{d}}$ do not depend on $R$, a given DLO with double exponential $\Sigma^{\text {mass }}(R)$ profile characterized by values of $R_{\mathrm{b}}, R_{\mathrm{d}}, \Sigma_{\mathrm{b}}^{\text {mass }}(0)$ and $\Sigma_{\mathrm{d}}^{\text {mass }}(0)$, would have a surface brightness profile, $\Sigma^{\text {light }}(R)$, given by Eqs. (2) and (3) with the same $R_{\mathrm{b}}$ and $R_{\mathrm{d}}$ parameters, but with $\Sigma_{\mathrm{b}}^{\text {light }}(0)=\frac{\Sigma_{\mathrm{b}}^{\text {mass }}(0)}{\Upsilon_{\mathrm{b}}}$ and $\Sigma_{\mathrm{d}}^{\text {light }}(0)=\frac{\Sigma_{\mathrm{d}}^{\text {mass }}(0)}{\Upsilon_{\mathrm{d}}}$. The previous comparisons will make sense if fittings to Monte Carlo realizations of a given $\Sigma^{\text {mass }}(R)$ profile and to Monte Carlo realizations of its surface brightness counterpart, $\Sigma^{\text {light }}(R)$, with the same number of particles, lead to the same values of the $R_{\mathrm{b}}$ and $R_{\mathrm{d}}$ parameters. This test has been performed for $\sum^{\text {mass }}(R)$ corresponding to DLOs \#242 and \#545 (from S1) in the sample. Results are shown in Fig. 5 for $\frac{\Upsilon_{\mathrm{b}}}{\Upsilon_{\mathrm{d}}}=1,3$ and 5 , from where we see that consistent values of $R_{\mathrm{b}}$ and $R_{\mathrm{d}}$ are obtained (within errors resulting from finite DLO sampling). Therefore, the mass and length structural parameters are decoupled, making the comparisons valid.

This compatibility between the simulated mass scale lengths and observed luminosity scale lengths in the $r$ and $K$ bands, suggests that the projected light density (i.e., stars) follows the projected mass density. This could be expected if the star formation rate in a disc were proportional to the baryon surface mass density (see Kennicutt 1998 and Silk 1999 for a discussion).

The mass $B / D$ ratios given in Table 2 are plotted in Fig. 6 versus the corresponding $n_{\text {opt }}$. Note that no correlation is observed between $B / D$ and $n_{\mathrm{opt}}$. These mass $B / D$ ratios are considerably higher than the corresponding luminosity $B / D$ ratios of late-type systems. But it is difficult to decide at the present whether the central baryon concentrations found in DLOs are excessive or not, as both, disc and bulge, mass-to-light ratios, would be required to compare with observed luminosity $B / D$ ratios. In fact, the only disc galaxy where the

(C) 0000 RAS, MNRAS 000, 000-000 
masses of the disc and bulge/central component can be estimated directly is the Milky Way. Sommer-Larsen \& Dolgov (1999) obtain $B / D \simeq 0.2-0.4$, for a disc scale length of $2.5-$ $3.0 \mathrm{kpc}$. For other galaxies, some estimations give $\Upsilon_{\mathrm{d}} \approx 1-2$ (in solar units, for $h=0.65$; Courteau \& Rix 1999, hereafter CR99). Values in these ranges would make the mass $B / D$ ratios obtained in the simulations roughly compatible with luminosity $B / D$ ratios from observations, but more accurate measures of $\Upsilon_{b}$ and $\Upsilon_{\mathrm{d}}$ are required before a conclusion can be reached. It is worthwhile to note that decompositions of extended H r rotation curves (RCs) of some galaxies from Broeils' compilation (B92), using sub-maximal discs (Bottema 1993, 1997; CR99), yield mass B / D ratios of about 1 (see Rhee 1996). Also, MGH99 find that 5 out of $23 \mathrm{Sa}-\mathrm{Scd}$ galaxies for which they obtain a reliable fit to the rotation curve (RC) for both the bulge and the disc component, have mass $B / D$ ratios of about 1 or larger. Note, however, that the majority of these decompositions are ill-constrained, as different combinations of bulge, disc, and halo profiles yield equally good agreement with the data (van Albada et al. 1985, CR99).

Current observational uncertainties on $\Upsilon_{\mathrm{b}}$ and $\Upsilon_{\mathrm{d}}$ preclude any consistency check for our derived baryon mass distributions. For more stringent constraints, one must turn instead to dynamical observables such as RCs.

\section{DLO ROTATION CURVES}

The shapes of spiral RCs are determined by their three dimensional total (i.e., bulge, disc and dark matter halo) mass distribution.

The RCs of the simulated objects have been constructed by adding up in quadrature the contributions to circular rotation from the bulge, the disc (both of them formed by baryons, either gas or stars, see $\left.\S_{3}\right)$, so that $V_{\mathrm{bar}}^{2}(r)=V_{\mathrm{b}}^{2}(r)+V_{\mathrm{d}}^{2}(r)$, and the dark matter halo, $V_{\mathrm{dm}}(r)$

$V_{\text {cir }}^{2}(r)=V_{\text {bar }}^{2}(r)+V_{\mathrm{dm}}^{2}(r)$.

We have adopted a softened Plummer potential (Evrard et al. 1994)

$V_{\mathrm{i}}^{2}(r)=\frac{G M_{\mathrm{i}}(<r) r^{2}}{\left(r^{2}+\epsilon_{\mathrm{g}}^{2}\right)^{3 / 2}}, \quad \mathrm{i}=$ bar, $\mathrm{dm}$

where $M_{\mathrm{bar}}(<r)$ and $M_{\mathrm{dm}}(<r)$ are the total baryonic and dark matter masses, respectively, inside a sphere or radius $r$. The adaptive AP3M scheme produces an effective dynamical gravitational softening at $z=0$ of $\epsilon_{\mathrm{g}}=3 \mathrm{kpc}$ at the central region of the DLOs. The RCs for several DLOs in our sample are given in Fig. 7. Points are the tangential velocity component of the baryonic particles (projected on the disc plane), solid lines are $V_{\text {cir }}(r)$, dashed lines are $V_{\mathrm{dm}}(r)$ and point lines $V_{\mathrm{bar}}(r)$. Note that the halo component, $V_{\mathrm{dm}}(r)$, gives rise to an almost flat contribution to the RCs beyond the very inner regions. These RCs fit very well the tangential velocity component of the baryonic particles up to $R \sim R_{\max }$, suggesting that these particles are in rotationally supported equilibrium within the potential well produced by both the dark haloes and the baryons themselves (TDT98).

On scales encompassing the baryonic object (i.e., $\leq R_{\max }$ ), DLOs have RCs that are declining in their outer parts, and some of them have central spikes produced by the bulge, similar to those found in some observed galaxies with mass $B / D \simeq 1$ or larger (for example, NGC 6674 and NGC 7331 in Rhee 1996, or UGC 89, UGC 1013 and UGC 1238 in MGH99). It is customary to try to explain the shapes of RCs as a combination of various 'fundamental plane' parameters such as global size (i.e., a spatial and a mass scale; Rubin et al. 1985), 
central mass concentration (Rhee \& van Albada 1996), and luminosity (Persic, Salucci \& Stel 1996). Here, the RC shapes for simulated DLOs are parametrized as a function of disc scale length, $R_{\mathrm{d}}$, as spatial scale, rotation velocity at $R_{2.2}=2.2 R_{\mathrm{d}}$ 开, $V_{2.2}=V_{\text {cir }}\left(R_{2.2}\right)$, as mass size, and central mass concentration, via the maximum or peak rotation velocity, $V_{\text {cir }}^{\text {peak }}$, and the radius where this is reached, $R_{\text {peak }}(\mathrm{C} 97 \mathrm{~b})$. Some combinations of these parameters have also been explored, such as the logarithmic slope, $L S$ (Casertano \& van Gorkom 1991 [CvG91]; B92; de Blok, McGaugh \& van der Hulst 1996). The $L S$ is observationally defined as the slope of a straight line that fits the $\mathrm{RC}$ in $\log -\log$ scale, from $R_{2.2}$ to $R_{\text {out }}$ (this last point is usually taken to be the last measured $\mathrm{RC}$ point, but see $\mathrm{C} 97 \mathrm{~b}$ for variations on this operational procedure). For simulated objects in our sample, the RCs are nearly linear in log - $\log$ scale for $R>R_{2.2}$, so that $L S$ does not appreciably change for $R_{\text {out }}>R_{2.2}$. The values of these parameters measured in simulated DLOs have been compared with those measured in RCs from: a) Sb-Sc field spirals drawn from the Courteau sample, with long-slit $\mathrm{H} \alpha$ spectra and $r$-band photometry (see C97b), and b) Sb-Sc spirals from Broeils' compilation (B92) of extended H i RCs with surface photometry. We keep only galaxies with $V_{2.2}>150$ $\mathrm{km} \mathrm{s}^{-1}$ (only one of Broeils' galaxies has $V_{2.2}$ in the range $[150,180] \mathrm{km} \mathrm{s}^{-1}$ ).

Apart from these parameters characterizing the shapes of RCs at scales of the baryonic objects, other interesting parameters at halo scales (i.e., $\sim r_{200}$ ), are the velocity at the virial radius $V_{200}=V_{\text {cir }}\left(r_{200}\right)$ and the spin parameter, $\lambda=\frac{J_{\text {tot }}\left|E_{\text {tot }}\right|^{1 / 2}}{G M_{\text {tot }}^{5 / 2}}$, with $J_{\text {tot }}, E_{\text {tot }}$ and $M_{\text {tot }}$ the total angular momentum, energy and mass for all particles inside $r_{200}$. Note that both $V_{200}$ and $\lambda$ are not observable.

In Table 3 we give $V_{200}, \lambda$ and the RC parameters for our DLO sample. Note that the $\lambda$ values are within their expected range for a standard CDM scenario (Warren et al. 1992; Dalcanton et al. 1997; Lemson \& Kauffmann 1998). In Fig. 8 we plot $R_{\mathrm{d}}$ versus $\lambda r_{200}$. A correlation appears in the sense that haloes with higher spin host more extended discs. In the framework of semianalytical models of quiescent pure (i.e., bulgeless) disc formation with $\boldsymbol{j}$ conservation one approximately has $R_{\mathrm{d}} \propto \lambda r_{200}$ (see Dalcanton et al. 1997; Mo et al. 1998); we find an important dispersion due to the loss of angular momentum by bulge particles involved in DAMCs in the violent phases of the evolution. The DLO values of the parameters $R_{\mathrm{d}}$ and $V_{2.2}$ are consistent with observations (see Tables 2 and 3 and Figs. 14 and 9). By virtue of selecting DLOs with $N_{\text {baryon }}>150$, we find high RC amplitudes mostly with $V_{2.2}>150$ $\mathrm{km} \mathrm{s}^{-1}$. Our simulated DLOs are either intermediate spirals $\left(100 \mathrm{~km} \mathrm{~s}^{-1}<V_{2.2}<180\right.$ $\mathrm{km} \mathrm{s}^{-1}, 9$ objects), large bright spirals $\left(V_{2.2}>180 \mathrm{~km} \mathrm{~s}^{-1}, R_{\mathrm{d}}>5.25 \mathrm{kpc}, 18\right.$ objects $)$, or compact bright spirals $\left(V_{2.2}>180 \mathrm{~km} \mathrm{~s}^{-1}, R_{\mathrm{d}}<5.25 \mathrm{kpc}, 2\right.$ objects). Our sample does not include any dwarf galaxy. As expected, $V_{2.2}$ is correlated with $V_{200}$, albeit with a dispersion. This dispersion is provided by the particularities of the evolutionary history of each DLO in the sample, namely, the number and characteristics of the interactions and merger events involved in its assembly (see TDT98 for a discussion).

The mass distribution of real spirals and their decomposition into bulge, disc and halo components cannot be inferred uniquely from observed RCs, as different decompositions yield equally good agreement with the data (van Albada et al. 1985; Broeils \& Courteau 1997; CR99). One has to postulate different scalings (mass-to-light ratios) between the bulge and disc components in order to infer the mass distribution of the halo. Common constraints for $(\mathrm{M} / \mathrm{L})_{\text {disc }}$ include a) the so-called 'maximum disc (or maximal light) hypothesis' (van Albada \& Sancisi 1986), which postulates that the halo dark mass component needed to fit the RCs should be minimum, or, equivalently, that the contribution of the disc and bulge

I $R_{2.2}$ is the radius at which a purely exponential disc would reach its maximum circular velocity.

(C) 0000 RAS, MNRAS 000, 000-000 
to the inner parts of RCs should be maximum, and b) sub-maximal discs with $\left(\frac{V_{\text {lum }}}{V_{\text {cir }}}\right)_{R_{2.2}} \sim$ $0.60 \pm 0.10$. This constraint is obtained either by matching the vertical velocity dispersion, scale height and scale length of a thin exponential disc which yields the maximum disc rotation (Bottema 1993), or, independently, showing that the Tully-Fisher relation of bright spirals is independent of surface brightness (CR99).

A maximum disc is defined as $\left(\frac{V_{\text {lum }}}{V_{\text {cir }}}\right)_{R_{2.2}}=0.85 \pm 0.10$, at the 95 per cent confidence level (Sackett 1997), corresponding to $\left(\frac{M_{\mathrm{lum}}}{M_{\mathrm{cir}}}\right)_{R_{2.2}}=0.72 \pm 0.17$. To the sub-maximal discs described above corresponds a $\left(\frac{M_{\text {lum }}}{M_{\text {cir }}}\right)_{R_{2 .}}$ of $0.36 \pm 0.12$. One notes that this choice of submaximal disc corresponds to half the amount of luminous matter at $R_{2.2}$ compared to the maximum disc case.

Let us now consider our simulations. $V_{\text {lum }, 2.2}=V_{\text {lum }}\left(R_{2.2}\right)$ can be measured directly once $R_{\mathrm{d}}$ is determined for each DLO (assuming that $V_{\text {lum }}=V_{\mathrm{bar}}$ ) and the different hypotheses above can be tested. Results are given in Table 3 for both the optimal and double exponential fits. $V_{\mathrm{bar}, 2.2}$ is correlated with $V_{2.2}$ (and, also, with $V_{200}$, albeit with more dispersion). In Fig. 10 we plot the $\left(\frac{V_{\text {bar }}}{V_{\text {cir }}}\right)_{R_{2.2}}$ ratios versus $V_{200}$ for DLOs in our sample (double exponential fits; no substantial changes occur taking instead the values corresponding to optimal fits). The shaded area shows the 95 per cent interval for maximum discs according to Sackett (1997). Only DLOs \#126 and \#545 (from S1) are inside this area, as they have $\left(\frac{V_{\text {bar }}}{V_{\text {cir }}}\right)_{R_{2.2}}=$ 0.765 and 0.763, respectively. These DLOs are somewhat peculiar: DLO \#126 has a particularly populated disc; DLO \#545 has two satellites close to it. We see that most DLOs lie inside the allowed interval for sub-maximal discs. The mean value for the simulated DLOs is $\left(\frac{V_{\text {bar }}}{V_{\text {cir }}}\right)_{R_{2.2}}=0.67 \pm 0.07$, in excellent agreement with Bottema $(1993,1997)$ and CR99.

In Fig. 10 only a slight correlation appears between $\left(\frac{V_{\text {bar }}}{V_{\text {cir }}}\right)_{R_{2.2}}$ and $V_{200}$ with an important dispersion, in the sense that less massive haloes have slightly lower values of $\left(\frac{V_{\mathrm{bar}}}{V_{\text {cir }}}\right)_{R_{2.2}}$. This presumably reflects the fact that less massive haloes are somewhat more centrally concentrated than more massive ones (Navarro, Frenk \& White 1995b; see also TDT98). The dispersion, on the other hand, reflects the particularities of the assembly of each DLO in the sample.

To quantify the importance of dark matter (or baryons) in the inner regions of DLOs we have used the $r_{\text {cross }}$ parameter (TDT98). We define $r_{\text {cross }}$ as the radius where $V_{\text {bar }}(r)=$ $V_{\mathrm{dm}}(r)$, such that RCs are dynamically dominated by baryons for $r<r_{\text {cross }}$, and dark matter dominated $r>r_{\text {cross }}$ (see Table 3). Note that most DLOs have $1 \leq \frac{r_{\text {cross }}}{R_{\mathrm{d}}} \leq 3$ and only a few have $r_{\text {cross }}>R_{2.2}$; note also that there is no correlation between $r_{\text {cross }}$ and $R_{\mathrm{d}}$. In Fig. 11 we plot $\frac{r_{\text {cross }}}{r_{200}}$ versus $V_{2.2}$; some correlation appears, in the sense that more massive objects are less dark matter dominated at their centres. This correlation disappears when $V_{2.2}$ is normalized to the halo global velocity, $V_{200}$, indicating that most of the correlation seen in Fig. 11 is provided by the halo total mass. In fact, as stated above, less massive haloes are known to be more centrally concentrated than more massive ones.

In Figs. 12(a) and 12(b), we plot $L S$ versus the disc scale lengths $R_{\mathrm{d}}$ and versus $V_{2.2}$, respectively, for the DLOs and, as a comparison, the objects of CvG91 compilation in the same dynamical range. We see that DLOs with $V_{2.2}>180 \mathrm{~km} \mathrm{~s}^{-1}$ do have observational counterparts, i.e., their $L S$ parameters take values that are found in observed spirals with the same range in $V_{2.2}$, even if we miss objects with $L S$ between, say, 0 and -0.1 . Those with $V_{2.2}<180 \mathrm{~km} \mathrm{~s}^{-1}$ have excessively low $L S \mathrm{~s}$, but recall that only one galaxy in CvG91 has $V_{2.2} \in[150,180] \mathrm{km} \mathrm{s}^{-1}$. Moreover, according to CvG91, LSs are correlated with the 
morphological type. Our DLOs would be $T \leq 5$, in consistence with the range in $T$ type previously found from the bulge shape parameters.

It is worthwhile to note that the value of the $L S$ is almost independent on how baryonic mass is distributed inside $R_{2.2}$, particularly on how it is shared between the bulge and the disc (it changes on average by only 10 per cent when the bulge mass is halved conserving the baryon number inside $R_{2.2}$ ). The fact that $L S$ s are negative may reflect in part the fact that dark matter haloes in standard CDM scenarios are too concentrated, leading to declining rotation curves (Navarro, Frenk \& White 1998). In fact, the declining shape of DLO RCs results from the flat halo contribution to RCs (see TDT98 for a detailed discussion) and the dynamic baryon dominance in the inner DLO regions. Declining RCs have been observed in spiral galaxies. Early examples can be found in Bosma (1978, 1981), van Moorsel (1982). Then, CvG91, B92 and Bosma (1999), among others, also found other examples. CvG91 suggest that this is a common feature of compact bright spiral galaxies, and also frequent in large bright spirals (although see B92 who shows, contrary to CvG91, that declining RCs do not correlate with the disc scale length). Dubinski, Mihos \& Hernquist (1999) have found that the declining character of RCs is a necessary condition to form long tidal tails in galaxy mergers as those observed in some interacting galaxy systems.

In Fig. 13 we plot $V_{\text {cir }}^{\text {peak }}$ versus the $R_{\text {peak }} / R_{\mathrm{d}}$ ratio for the 29 DLOs in our sample. For comparison, we also give the peak velocities in $\mathrm{H}$ I of $\mathrm{Sb}-\mathrm{Sc}$ spirals from table 4.1 in RC96 (most of them from B92 compilation), with $R_{\text {peak }} / R_{\mathrm{d}}$ ratios taken from C97b. We also plot the $V_{\text {cir }}^{\text {peak }}$ and $R_{\text {peak }} / R_{\text {d }}$ ratios measured in the Courteau sample of optical RCs (C97b). We confirm the previous finding that DLOs in Table 1 have observational counterparts, even if the smaller ones are somewhat too concentrated as compared with Courteau data. Note, however, that $R_{\text {peak }}$ is difficult to measure in DLOs and the measurement hard to assess in spiral galaxies.

\section{SUMMARY AND CONCLUSIONS}

We present the results of a detailed and careful comparison between the parameters characterizing the structural and dynamical properties of a sample of 29 simulated DLOs and those measured in observed spiral galaxies. These DLOs have been identified in three fully consistent hierarchical hydrodynamical simulations, where an inefficient Schmidt law-like algorithm to model the stellar formation process has been implemented. In this paper we have been only concerned with disc structural and dynamical properties, because they keep the imprints of the dynamical and hydrodynamical processes that, together with their interplay with star formation, are key for galaxy formation and evolution.

The comparison of DLOs in our sample with spiral discs has been a two step work. First, DLO bulge-disc decomposition has been performed. This yields the bulge effective scale length and shape parameters, $R_{\mathrm{b} \text {,eff }}$ and $n_{\mathrm{opt}}$, the disc scale length, $R_{\mathrm{d}}$, and the mass bulge-to-disc ratio, $B / D$, among other DLO parameters. After having tested the robustness of the fitting procedure, the following results have been obtained:

(i) The scale lengths, $R_{\mathrm{b} \text {,eff }}$ and $R_{\mathrm{d}}$, and their ratio $R_{\mathrm{b}, \text { eff }} / R_{\mathrm{d}}$, are consistent with available data (CdJB96; de Jong 1996; MGH99).

(ii) The distribution of bulge shape parameters is similar to that found by CdJB96 for their sample of $\mathrm{Sa}-\mathrm{Sc}$ galaxies.

(iii) The mass $B / D$ ratios are somewhat high as compared to luminosity $B / D$ ratios, but current observational uncertainties on the bulge and disc mass-to-light ratios make it difficult to draw any conclusion from this comparison.

(C) 0000 RAS, MNRAS 000, 000-000 
In a second step, DLO RCs have been analyzed. DLO gas particles placed at distances between $2 \mathrm{kpc}$ and $30 \mathrm{kpc}$ to the centre move roughly along circular orbits on the equatorial plane. They are in centrifugal supported equilibrium within the potential well produced by the total mass distribution (TDT98). Rotation curve shapes have been parametrized through $R_{\mathrm{d}}$ (or $R_{2.2}=2.2 R_{\mathrm{d}}$ ) as spatial scale, $V_{2.2}=V_{\text {cir }}\left(R_{2.2}\right)$ as mass size, and the maximum or peak velocity, $V_{\text {cir }}^{\text {peak }}$, and the radius where this is reached, $R_{\text {peak }}$. These parameters have been compared with those measured at: a) $\mathrm{Sb}-\mathrm{Sc}$ field spirals drawn from the Courteau sample, with long-slit $\mathrm{H} \alpha$ spectrum and $r$-band photometry (C97b), and b) Sb-Sc spirals from Broeils' compilation (B92) of extended H I RCs with surface photometry. The main results follow:

(i) In contrast to findings in other fully-consistent hydrodynamical simulations (e.g., Navarro \& Steinmetz 2000; Thacker \& Couchman 2000), DLO $V_{2.2}$ velocities have been found to be consistent with observational data. This is a consequence of disc formation with $j$ conservation.

(ii) The $R_{\mathrm{d}}$ and $V_{2.2}$ values obtained indicate that DLOs in our sample are either large bright spirals (18), intermediate spirals (9), or compact bright spirals (2).

(iii) The average relative contribution of baryons to $V_{2.2}$ in our sample is $\left(\frac{V_{\text {bar }}}{V_{\text {cir }}}\right)_{R_{2.2}}=$ $0.67 \pm 0.07$, in very good agreement with Bottema $(1993,1997)$ and CR99, if we take $V_{\text {bar }}=$ $V_{\text {lum. }}$. Most DLOs have been found to have sub-maximal discs; only two of them have been found to lie inside the 95 per cent confidence interval for maximum discs according to Sackett (1997). The previous agreement also shows that the amount of baryon mass that has ended up inside $R<R_{2.2}$ is not excessive, again as a consequence of $\boldsymbol{j}$ conservation.

(iv) The $\frac{r_{\text {cross }}}{r_{200}}$ parameter ratio is a measure of the relative amount of dark matter at the inner DLO zones. Some correlation between these amounts and the circular velocity at $R_{2.2}$ has been found, in the sense that less massive objects tend to be more dark matter dominated in their central regions.

(v) Concerning the parameters that give a measure of the central concentrations, the comparison of the $V_{\text {cir }}^{\text {peak }}$ versus $\frac{R_{\text {peak }}}{R_{\mathrm{d}}}$ plots to B92 data confirms again that DLOs have observational counterparts, even if the smaller DLOs are somewhat too concentrated as compared to Courteau data (C97b).

(vi) The logarithmic slope, $L S$, measures the $\mathrm{RC}$ slopes for $R>R_{2.2}$, where, as stated, in most cases dark matter is already dynamically dominant. DLOs with $V_{2.2}>180 \mathrm{~km} \mathrm{~s}^{-1}$ occupy the same zone in the $L S$ versus $V_{2.2}$ and versus $R_{2.2}$ plots as galaxies studied by CvG91 in the same dynamical range; those with $V_{2.2}<180 \mathrm{~km} \mathrm{~s}^{-1}$ do not show the tendency of less massive galaxies in CvG91 to have higher $L S$. This may be a consequence of the strongly concentrated mass distribution of dark haloes in standard CDM scenarios.

A concern is in order regarding numerical resolution. DLOs are resolved with a relatively low number of particles. In contrast, dark matter haloes are described with a much better resolution. An inappropriate low gas resolution would result in an unphysical gas heating that could halt the gas collapse (Navarro \& Steinmetz 1997). However, some works suggest that it is an inadequate resolution in the dark matter halo component that may produce the larger undesired numerical artifacts (Steinmetz \& White 1997). In fact, it appears that a well-resolved dark matter halo, even if the number of gas particles is lower (but more than, say, one hundred), gives rise to a well-represented (or well-resolved) gas density profile, being this point the most important for both the hydrodynamics and the tracking of the star formation history. Moreover, to make sure that the populated and extended discs in the simulations do not result from unphysical gas heating or smoothing, we have run a 
higher resolution simulation $\left(64^{3}\right.$ particles in a periodic box of $5 \mathrm{Mpc}$, with cosmological and star formation parameters similar to those in S1, S1b and S1c; hereafter HRS). Only one disc with mass comparable to those in these lower resolution simulations forms. It has 1713+1380 gas+star particles and its halo 36112 dark matter particles. Its analysis has shown that it is populated and extended, that its structural and dynamical characteristics are also compatible with observations (see table 1 and fig. 1 in Domínguez-Tenreiro et al. 1998, where we show that the $R_{\mathrm{b}}$ and $R_{\mathrm{d}}$ parameters and their ratio take a typical value, and the specific angular momentum has been conserved) and that the physical processes leading to its formation are essentially the same as those that are at work in S1, S1b or S1c.

In conclusion, the comparison between DLOs produced in our simulations and observational data allows us to affirm that they have counterparts in the real world. This agreement suggests that the process operating in Fall \& Efstathiou (1980) standard model for disc formation (i.e., gas cooling and collapse with specific angular momentum, $\boldsymbol{j}$, conservation) is also at work in the quiescent phases of DLO formation in these simulations, resulting in discs with exponential density profiles, as predicted by Dalcanton et al. (1997). However, in Domínguez-Tenreiro et al. (1998) and Sáiz et al. (1999) it is shown that violent episodes (i.e., interaction and merger events) also occur and play an important role in DLO assembly. In particular, it is shown that dark matter haloes formed in these simulations are not always able to stabilize the pure exponential disc that would form at its central region according to Fall \& Efstathiou and Dalcanton et al. scenarios (see also van den Bosch 2000). To provide the right conditions for disc regeneration to occur after the last violent episode of DLO assembly, a compact central bulge is needed; this will ensure the axisymmetric character of the gravitational potential well at scales of some kpcs at all times, avoiding excessive $\boldsymbol{j}$ losses in violent events. In TSDT these arguments are developed, and, moreover, we prove that a second condition is necessary for disc regeneration: the availability of gas at low $z$, that is, it is necessary that the SF algorithm implementation does not result in a too early gas exhaustion. The good behaviour of our DLO sample as compared with observations suggests that the inefficient SF algorithm used in the simulations has met both requirements.

The global agreement with observations we have found, at a structural and dynamical level, also represents an important step towards making numerical approaches more widely used to learn about galaxy formation and evolution in a cosmological framework, i.e., from primordial fluctuations. These approaches will be particularly useful if, as expected, future improvements in numerical methods and computers speed allow to numerically simulate the different aspects of galaxy assembly with an increasingly high degree of realism.

\section{ACKNOWLEDGMENTS}

It is a pleasure to thank E. Bertschinger for providing us with some software subroutines, J. F. Navarro for interesting discussions and J. Silk for his valuable comments on our work. We wish to acknowledge as well the referee, J. Sommer-Larsen, for his suggestions and interesting comments. This work was supported in part by DGES (Spain) through grants number PB93-0252 and PB96-0029. AS was also supported by DGES through fellowships. PBT thanks DGES, and CONICET and ANPCyT (Argentina) for their financial support. We are indebted to the Centro de Computación Científica (Universidad Autónoma de Madrid) and to the Oxford University for providing the computational support to perform this work.

(C) 0000 RAS, MNRAS 000, 000-000 


\section{REFERENCES}

Andredakis Y.C., Peletier R.F., Balcells M., 1995, MNRAS, 275, 874

Athanassoula E., Sellwood J.A., 1986, MNRAS, 221, 213

Barnes J.E., Hernquist L., 1991, ApJ, 370, L65; 1992, ARA\&A, 30, 705

Binney J., Tremaine S., 1987, Galactic Dynamics. Princeton Univ. Press, Princeton, NJ

Bosma A., 1978, Ph.D. thesis, Univ. Groningen

Bosma A., 1981, AJ, 86, 1791

Bosma A., 1999, in Merritt D.R., Valluri M., Sellwood J.A., eds, ASP Conf. Ser. Vol. 182, Galaxy Dynamics - A Rutgers Symposium. Astron. Soc. Pac., San Francisco, p. 339

Bottema R., 1993, A\&A, 275, 16

Bottema R., 1997, A\&A, 328, 517

Broeils A.H., 1992, Ph.D. thesis, Univ. Groningen (B92)

Broeils A.H., Courteau S., 1997, in Persic M., Salucci P., eds, ASP Conf. Ser. Vol. 117, Dark and Visible Matter in Galaxies and Cosmological Implications. Astron. Soc. Pac., San Francisco, p. 74

Casertano S., van Gorkom J.H., 1991, AJ, 101, 1231 (CvG91)

Courteau S., 1996, ApJS, 103, 363

Courteau S., 1997a, in Block D., Greenberg M., eds, Morphology \& Dust Content in Spiral Galaxies. Kluwer, Dordrecht

Courteau S., 1997b, AJ, 114, 2402 (C97b)

Courteau S., Rix H.-W., 1999, ApJ, 513, 561 (CR99)

Courteau S., de Jong R.S., Broeils, A.H., 1996, ApJ, 457, L73 (CdJB96)

Christodoulou D.M., Shlosman I., Tohline J.E., 1995, ApJ, 443, 551

Dalcanton J.J., Spergel D.N., Summers F.J., 1997, ApJ, 482, 659

Dalgarno A., McCray R.A., 1972, ARA\&A, 10, 375

de Blok W.J.G., McGaugh S.S., van der Hulst J.M., 1996, MNRAS, 283, 18

de Jong R.S., 1996, A\&A, 313, 45

Domínguez-Tenreiro R., Tissera P.B., Sáiz A., 1998, ApJ, 508, L123

Dubinski J., Mihos J.C., Hernquist L., 1999, ApJ, 526, 607

Evrard A.E., Summers F.J., Davis M., 1994, ApJ, 422, 11

Fall S.M., 1983, in Athanassoula E., ed., IUA Symp. 100, Internal Kinematics and Dynamics of Galaxies. Reidel, Dordrecht, p. 391

Fall S.M., Efstathiou G., 1980, MNRAS, 193, 189

Firmani C., Avila-Reese V., 2000, MNRAS, 315, 457

Katz N., 1992, ApJ, 391, 502

Kennicutt R.C. Jr., 1998, ApJ, 498, 541

Lemson G., Kauffmann G., 1998, MNRAS, 302, 111

Martinet L., 1995, Fund. Cosmic Phys., 15, 341

Mihos J.C., Hernquist L., 1994, ApJ, 425, L13; 1996, ApJ, 464, 641

Mo H.J., Mao S., White S.D.M., 1998, MNRAS, 295, 319

Moriondo G., Giovanelli R., Haynes M.P., 1999, A\&A, 346, 415 (MGH99)

Navarro J.F., Benz, W., 1991, ApJ, 380, 320

Navarro J.F., Steinmetz M., 1997, ApJ, 478, 13

Navarro J.F., Steinmetz M., 2000, ApJ, 538, 477

Navarro J.F., White S.D.M., 1993, MNRAS, 265, 271

Navarro J.F., Frenk C.S., White S.D.M., 1995a, MNRAS, 275, 56; 1995b, MNRAS, 275, 720

Persic M., Salucci P., Stel F., 1996, MNRAS, 281, 27

Pohlen M., Dettmar R.-J., Lütticke R., 2000, A\&A, 357, L1

Rhee M.H., 1996, Ph.D. thesis, Univ. Groningen

Rhee M.H., van Albada T.S., 1996, A\&AS, 115, 407

Rubin V.C., Burstein D., Ford W.K. Jr., Thonnard N., 1985, ApJ, 289, 81

Sackett P.D., 1997, ApJ, 483, 103

Sáiz A., Tissera P.B., Domínguez-Tenreiro R., 1999, Ap\&SS, 263, 43

Sérsic J.L., 1968, Atlas de Galaxias Australes (Observatorio Astronómico de Córdoba)

Silk J., 1999, in Hammer F., Thuan T.X., Cayatte V., Guiderdoni B., Tran Thanh Van J., eds, Proceedings of the XIXth Moriond Meeting. Ed. Frontières, in press

Sommer-Larsen J., Dolgov A., 1999, astro-ph/9912166 preprint

Sommer-Larsen J., Gelato S., Vedel H., 1999, ApJ, 519, 501

Steinmetz M., Müller E., 1995, MNRAS, 276, 549

Steinmetz M., Navarro J.F., 1999, ApJ, 513, 555

Steinmetz M., White, S.D.M., 1997, MNRAS, 289, 545

Thacker R.J., Couchman H.M.P., 2000, astro-ph/0001268 preprint

Thomas P.A., Couchman H.M.P., 1992, MNRAS, 257, 11

Tissera P.B., 2000, ApJ, in press

Tissera P.B., Domínguez-Tenreiro R., 1998, MNRAS, 297, 177 (TDT98)

Tissera P.B., Lambas D.G., Abadi M.G., 1997, MNRAS, 286, 384

van Albada T.S., Sancisi R., 1986, Phil. Trans. R. Soc. London Ser. A, 320, 447

van Albada T.S., Bahcall J.N., Begeman K., Sancisi R., 1985, ApJ, 295, 305

van den Bosch F.C., 1998, ApJ, 507, 601; 2000, ApJ, 530, 177 
van Moorsel G.A., 1982, A\&A, 107, 66

Vedel H., Hellsten U., Sommer-Larsen J., 1994, MNRAS, 271, 743

Warren M.S., Quinn P.J., Salmon J.K., Zurek W.H., 1992, ApJ, 399, 405

Weil M.L., Eke V.R., Efstathiou G., 1998, MNRAS, 300, 773 
Table 1. General Parameters

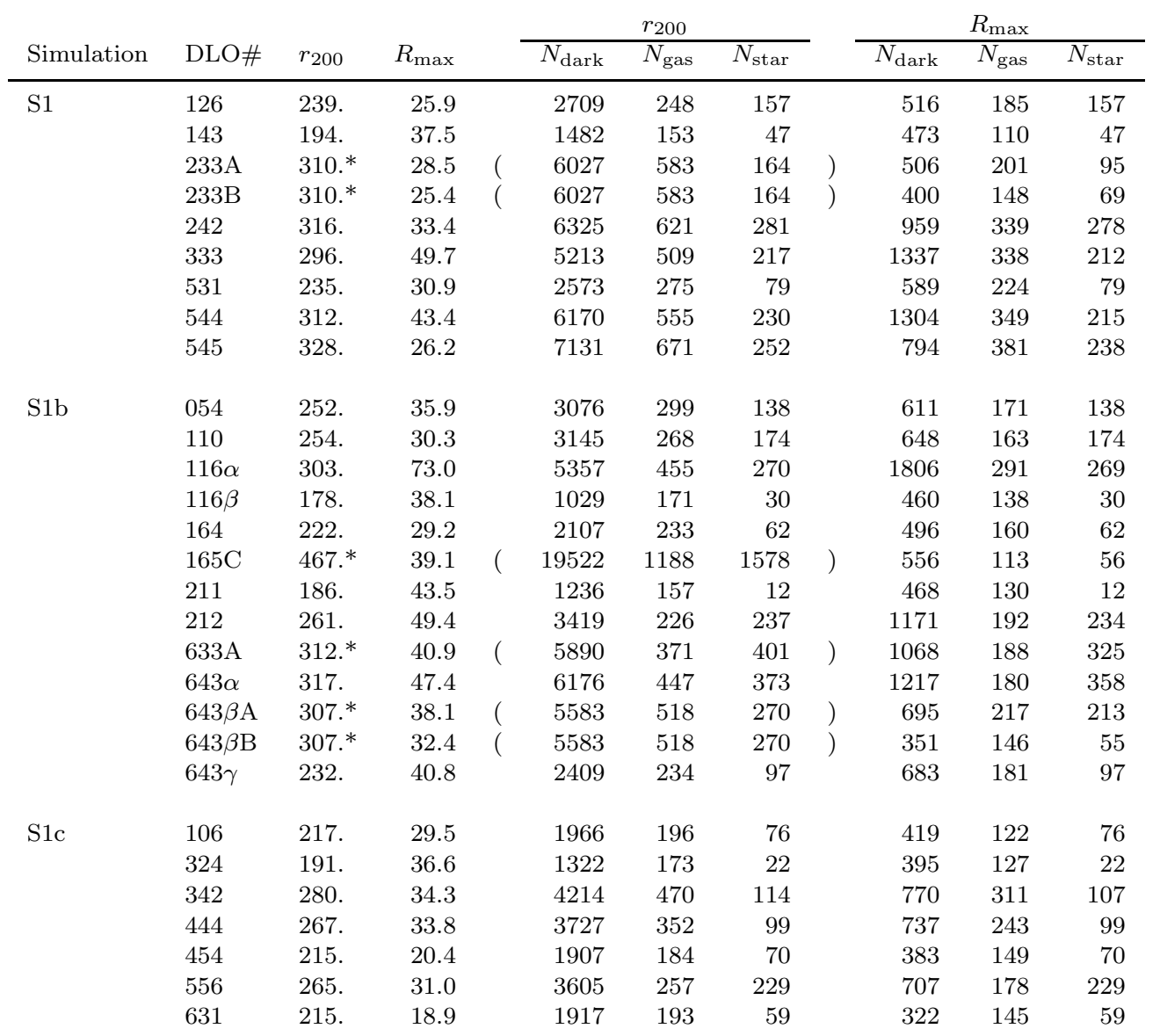

Distances are given in kpc. An asterisk indicates that the corresponding DLO is embedded in a double or multiple halo, i.e., that one or more other baryonic objects (either disc-like or not) exist within $r_{200}$. This is to be taken into account on evaluating the total number of halo particles (numbers in parentheses). 
Table 2. Bulge/disc parameters

\begin{tabular}{|c|c|c|c|c|c|c|c|c|c|c|c|c|c|}
\hline \multirow[b]{2}{*}{ DLO\# } & \multicolumn{6}{|c|}{ Optimal Fit } & \multicolumn{5}{|c|}{$n=1$} & \multicolumn{2}{|c|}{$n=4$} \\
\hline & $n_{\mathrm{opt}}$ & $R_{\mathrm{b}, \text { eff }}$ & $R_{\mathrm{d}}$ & $B / D$ & $\chi^{2} / N$ & $R_{\text {trans }}$ & $R_{\mathrm{b}, \text { eff }}$ & $R_{\mathrm{d}}$ & $B / D$ & $\chi^{2} / N$ & $R_{\text {trans }}$ & $\overline{R_{\mathrm{d}}}$ & $\chi^{2} / N$ \\
\hline 126 & 2.6 & 0.90 & 6.3 & 1.83 & 5.72 & 4.46 & 1.09 & 5.8 & 1.48 & 10.40 & 3.50 & 6.5 & 6.35 \\
\hline 143 & 3.2 & 0.58 & 10.9 & 1.45 & 1.61 & 4.54 & 0.91 & 10.1 & 1.36 & 2.30 & 3.52 & 11.1 & 1.53 \\
\hline $233 \mathrm{~A}$ & 1.3 & 1.00 & 6.5 & 1.25 & 5.52 & 3.57 & 1.08 & 6.4 & 1.22 & 5.55 & 3.43 & 7.1 & 5.81 \\
\hline $233 \mathrm{~B}$ & 0.6 & 1.05 & 5.6 & 1.22 & 9.09 & 2.77 & 0.89 & 5.7 & 1.23 & 9.55 & 2.89 & 5.8 & 12.54 \\
\hline 242 & 1.2 & 1.25 & 7.5 & 1.23 & 15.96 & 4.19 & 1.29 & 7.5 & 1.21 & 16.19 & 4.06 & 7.9 & 42.04 \\
\hline 333 & 1.9 & 1.36 & 12.0 & 1.72 & 5.30 & 6.59 & 1.50 & 11.1 & 1.58 & 11.40 & 5.36 & 12.8 & 11.98 \\
\hline 531 & 0.9 & 1.70 & 6.9 & 2.02 & 3.85 & 5.24 & 1.69 & 6.9 & 2.07 & 4.00 & 5.39 & 9.3 & 19.57 \\
\hline 544 & 2.3 & 1.05 & 10.6 & 1.79 & 2.44 & 5.82 & 1.26 & 9.7 & 1.61 & 9.05 & 4.54 & 11.3 & 4.06 \\
\hline 545 & 1.1 & 1.26 & 5.9 & 1.23 & 3.01 & 3.78 & 1.27 & 5.9 & 1.22 & 3.11 & 3.74 & 6.4 & 20.24 \\
\hline 054 & 1.6 & 0.89 & 8.4 & 2.99 & 2.94 & 4.80 & 1.04 & 8.0 & 2.82 & 3.65 & 4.13 & 9.7 & 4.32 \\
\hline 110 & 1.8 & 0.60 & 7.0 & 2.54 & 2.64 & 3.46 & 0.78 & 6.8 & 2.47 & 3.28 & 3.13 & 7.2 & 3.46 \\
\hline $116 \alpha$ & 2.3 & 0.48 & 16.4 & 1.40 & 13.97 & 4.12 & 0.76 & 16.3 & 1.39 & 14.55 & 3.50 & 16.5 & 14.79 \\
\hline $116 \beta$ & 1.3 & 1.26 & 8.7 & 1.70 & 1.75 & 4.97 & 1.34 & 8.5 & 1.64 & 1.93 & 4.60 & 10.1 & 3.10 \\
\hline 164 & 1.3 & 1.19 & 7.2 & 2.47 & 1.70 & 4.83 & 1.25 & 6.9 & 2.36 & 1.99 & 4.44 & 9.9 & 4.64 \\
\hline $165 \mathrm{C}$ & 1.6 & 0.93 & 9.0 & 2.37 & 1.08 & 4.67 & 1.06 & 8.7 & 2.28 & 1.28 & 4.15 & 9.8 & 1.71 \\
\hline 211 & 1.8 & 1.35 & 14.0 & 1.42 & 0.75 & 6.47 & 1.49 & 13.0 & 1.36 & 1.19 & 5.40 & 17.0 & 1.30 \\
\hline 212 & 2.3 & 0.53 & 11.2 & 1.95 & 4.93 & 4.04 & 0.80 & 11.1 & 1.91 & 6.30 & 3.46 & 11.3 & 5.49 \\
\hline $633 \mathrm{~A}$ & 1.1 & 0.72 & 9.0 & 3.01 & 4.69 & 3.33 & 0.74 & 9.2 & 2.98 & 4.33 & 3.32 & 9.6 & 6.62 \\
\hline $643 \alpha$ & 1.4 & 1.19 & 10.9 & 3.40 & 3.14 & 5.91 & 1.28 & 10.6 & 3.27 & 5.07 & 5.30 & 13.2 & 19.61 \\
\hline $643 \beta \mathrm{A}$ & 2.8 & 0.86 & 9.4 & 2.36 & 3.18 & 5.75 & 1.13 & 8.5 & 2.02 & 10.91 & 4.22 & 10.0 & 3.70 \\
\hline $643 \beta \mathrm{B}$ & 1.4 & 1.28 & 7.6 & 1.98 & 1.46 & 5.02 & 1.35 & 7.3 & 1.87 & 1.96 & 4.54 & 9.5 & 3.45 \\
\hline $643 \gamma$ & 1.7 & 1.18 & 13.2 & 2.43 & 2.90 & 6.56 & 1.32 & 11.9 & 2.32 & 4.33 & 5.30 & 17.8 & 5.37 \\
\hline 106 & 2.0 & 1.06 & 7.5 & 2.69 & 1.18 & 5.51 & 1.20 & 6.6 & 2.25 & 2.47 & 4.22 & 8.2 & 1.70 \\
\hline 324 & 1.2 & 1.42 & 8.3 & 1.96 & 0.80 & 5.22 & 1.46 & 8.2 & 1.91 & 0.84 & 4.99 & 10.1 & 2.56 \\
\hline 342 & 1.3 & 1.43 & 8.2 & 1.45 & 2.52 & 5.09 & 1.46 & 7.7 & 1.38 & 2.87 & 4.60 & 9.2 & 9.95 \\
\hline 444 & 1.3 & 1.41 & 7.9 & 2.02 & 3.49 & 5.34 & 1.46 & 7.6 & 1.92 & 4.22 & 4.89 & 10.9 & 11.14 \\
\hline 454 & 1.0 & 1.23 & 4.6 & 3.14 & 2.33 & 4.26 & 1.24 & 4.6 & 3.10 & 2.34 & 4.20 & 10.2 & 6.45 \\
\hline 556 & 2.1 & 0.86 & 7.6 & 3.70 & 1.64 & 5.37 & 1.06 & 6.9 & 3.17 & 6.17 & 4.13 & 8.2 & 3.70 \\
\hline 631 & 0.9 & 1.13 & 4.1 & 2.36 & 0.97 & 3.50 & 1.11 & 4.2 & 2.43 & 0.97 & 3.62 & 18.0 & 2.78 \\
\hline
\end{tabular}

Distances are given in kpc. 
Table 3. Dynamical Parameters

\begin{tabular}{|c|c|c|c|c|c|c|c|c|c|c|}
\hline \multirow[b]{2}{*}{ DLO\# } & \multirow[b]{2}{*}{$V_{200}$} & \multirow[b]{2}{*}{$\lambda$} & \multirow[b]{2}{*}{$r_{\text {cross }}$} & \multirow[b]{2}{*}{$L S$} & \multirow[b]{2}{*}{$R_{\text {peak }}$} & \multirow[b]{2}{*}{$V_{\text {cir }}^{\text {peak }}$} & \multicolumn{2}{|c|}{$n=1$} & \multicolumn{2}{|c|}{ Optimal Fit } \\
\hline & & & & & & & $V_{2.2}$ & $V_{\text {bar }, 2.2}$ & $V_{2.2}$ & $V_{\mathrm{bar}, 2.2}$ \\
\hline 126 & 114. & 0.039 & 16.6 & -0.176 & 10.6 & 216. & 210. & 157. & 206. & 153. \\
\hline 143 & 98.5 & 0.045 & 8.94 & -0.172 & 7.98 & 156. & 148. & 83.0 & 147. & 80.6 \\
\hline $233 \mathrm{~A}$ & $157 *^{*}$ & $0.063^{*}$ & 14.0 & -0.139 & 9.45 & 203. & 197. & 139. & 197. & 139. \\
\hline $233 \mathrm{~B}$ & $157 .^{*}$ & $0.063^{*}$ & 12.9 & -0.085 & 6.93 & 179. & 176. & 125. & 176. & 125. \\
\hline 242 & 160. & 0.069 & 19.3 & -0.168 & 6.33 & 279. & 257. & 186. & 257. & 186. \\
\hline 333 & 150. & 0.079 & 16.2 & -0.108 & 7.25 & 250. & 221. & 143. & 218. & 139. \\
\hline 531 & 118. & 0.011 & 12.7 & -0.192 & 7.70 & 217. & 206. & 140. & 206. & 140. \\
\hline 544 & 158. & 0.023 & 17.8 & -0.104 & 5.69 & 254. & 228. & 153. & 225 . & 148. \\
\hline 545 & 166. & 0.049 & 18.6 & -0.159 & 8.66 & 278. & 270 & 204. & 269. & 204. \\
\hline 054 & 126. & 0.122 & 16.4 & -0.152 & 5.64 & 213. & 189. & 132. & 187. & 129. \\
\hline 110 & 127. & 0.044 & 12.2 & -0.172 & 6.74 & 237 & 215. & 148. & 214. & 147. \\
\hline $116 \alpha$ & 151. & 0.067 & 16.8 & -0.097 & 6.46 & 249. & 206. & 121. & 206. & 121. \\
\hline $116 \beta$ & 88.8 & 0.055 & 8.80 & -0.214 & 8.11 & 167. & 155. & 92.6 & 153. & 92.0 \\
\hline 164 & 111. & 0.038 & 9.63 & -0.193 & 6.88 & 199. & 186. & 120. & 184. & 118. \\
\hline $165 \mathrm{C}$ & $228 .^{*}$ & $0.043^{*}$ & 7.70 & -0.136 & 7.43 & 176. & 160. & 92.7 & 160. & 92.6 \\
\hline 211 & 92.7 & 0.095 & 7.29 & -0.189 & 7.15 & 153. & 136. & 70.8 & 135. & 69.0 \\
\hline 212 & 130. & 0.062 & 13.2 & -0.155 & 5.64 & 243. & 213 & 132. & 213. & 131. \\
\hline $633 \mathrm{~A}$ & $156 .^{*}$ & $0.067^{*}$ & 19.9 & -0.161 & 5.78 & 265. & 227 & 160. & 227. & 161. \\
\hline $643 \alpha$ & 158. & 0.086 & 20.6 & -0.118 & 5.78 & 275. & 222 . & 153. & 222 . & 152. \\
\hline $643 \beta \mathrm{A}$ & $154 .^{*}$ & $0.067^{*}$ & 20.3 & -0.184 & 7.43 & 234. & 209. & 149. & 204. & 144. \\
\hline $643 \beta \mathrm{B}$ & $154 .^{*}$ & $0.067^{*}$ & 15.1 & -0.166 & 8.11 & 170. & 157. & 110 . & 156. & 108. \\
\hline $643 \gamma$ & 116. & 0.054 & 12.7 & -0.174 & 8.53 & 205. & 176. & 105. & 171. & 100. \\
\hline 106 & 108. & 0.075 & 12.4 & -0.167 & 5.64 & 177. & 169. & 116. & 167. & 110. \\
\hline 324 & 95.2 & 0.045 & 8.53 & -0.201 & 8.66 & 160. & 147. & 89.9 & 147. & 88.8 \\
\hline 342 & 140. & 0.018 & 15.1 & -0.152 & 9.76 & 235. & 220 . & 153. & 217. & 149. \\
\hline 444 & 133. & 0.055 & 14.0 & -0.135 & 6.74 & 225. & 207. & 141. & 207. & 139. \\
\hline 454 & 107. & 0.034 & 10.6 & -0.154 & 6.88 & 205. & 197. & 141. & 197. & 141. \\
\hline 556 & 130. & 0.063 & 15.4 & -0.167 & 6.46 & 248. & 226 . & 160. & 222 . & 155. \\
\hline 631 & 107. & 0.025 & 9.63 & -0.178 & 6.33 & 199. & 196. & 140 . & 196. & 141. \\
\hline
\end{tabular}

Distances are given in kpc; velocities in $\mathrm{km} \mathrm{s}^{-1}$. Asterisks stand for double or multiple haloes, so that their global parameters (with an asterisk in this Table) might not reflect a direct property of the corresponding DLO. 
Figure 1. Upper (a): The cosine of the angle formed by the position and velocity vectors of each baryon particle within halo \#242, versus their positions. Dots: gas particles, stars: stellar particles; open symbols: counter-rotating particles. Lower (b): Specific angular momentum component along $\boldsymbol{j}_{\text {dis }}$ for each baryon particle of halo \#242, versus their positions. Solid line: $V_{\text {cir }}(R) R$.

Figure 2. Specific angular momentum at $z=0$ versus the mass for the haloes (open symbols), for the inner 83 per cent of the gas component in the DLOs (filled symbols), and for their stellar component (starred symbols). Squares, triangles and circles correspond to DLOs formed in S1, S1b and S1c simulations, respectively. The solid (dotted) box encloses the region occupied by the spiral discs (ellipticals), as given by Fall (1983).

Figure 3. Thick lines: the histograms for the integrated projected mass density of baryons, $M^{\text {cyl }}\left(R_{i}\right)$, for DLOs \#242 and \#643 $\beta$ A (see Table 1). Thin lines are their best fits by means of a bulge-disc decomposition. A solid-line arrow marks $R_{\text {trans }}$ and a dashed-line arrow marks $R_{\text {min }}$.

Figure 4. The bulge and disc scale lengths, $R_{\mathrm{b}}$ and $R_{\mathrm{d}}$, for double exponential fits. Squares, triangles and circles correspond to DLOs formed in S1, S1b and S1c simulations, respectively. Dots are from 1D decompositions of surface brightness profiles given by CdJB96 and Courteau (1997a). The error bars in the top left corner are the typical dispersions in the $R_{\mathrm{b}}$ and $R_{\mathrm{d}}$ values for DLOs, obtained from Monte Carlo realizations of their profiles.

Figure 5. Average values of the $R_{\mathrm{b}}$ and $R_{\mathrm{d}}$ parameters and their dispersions, obtained from Monte Carlo realizations of DLOs \#242 and \#545 $\Sigma^{\text {light }}(R)$ profiles (see text). Thick lines correspond to $\frac{\Upsilon_{\mathrm{b}}}{\Upsilon_{\mathrm{d}}}=1$ (that is, $\Sigma^{\text {light }}(R) \propto \Sigma^{\text {mass }}(R)$ ), thin lines to $\frac{\Upsilon_{\mathrm{b}}}{\Upsilon_{\mathrm{d}}}=3$ and dotted lines to $\frac{\Upsilon_{\mathrm{b}}}{\Upsilon_{\mathrm{d}}}=5$.

Figure 6. The mass bulge-to-disc ratios $B / D$ versus the optimal bulge shape parameter, $n_{\mathrm{opt}}$, for the DLO sample. Squares, triangles and circles correspond to DLOs formed in S1, S1b and S1c simulations, respectively. 
Figure 7. Points: the rotational velocity distributions for the baryon particles of DLOs \# 242 of S1, \#212 and \#643ßA of S1b and \#342 of S1c (see text). Solid lines: circular velocity curves (see Eqs. (8) and (9)) for the total mass distribution; dotted lines: the baryonic contribution $V_{\mathrm{bar}}(r)$, and dashed lines: the dark halo contribution $V_{\mathrm{dm}}(r)$.

Figure 8. The disc scale length $R_{\mathrm{d}}$ versus $\lambda r_{200}$ for the DLO sample. Symbols are as in Fig. 6. DLOs belonging to haloes that include several objects (either DLOs, spheroids or irregulars) have not been considered.

Figure 9. The $V_{2.2}$ velocities versus the disc scale lengths for the DLO sample. Squares, triangles and circles correspond to DLOs formed in S1, S1b and S1c simulations, respectively. Dots are data from the Courteau sample (H $\alpha$ spectra, see C97b). Open circles are data from spirals with H i rotation curves from CvG91 and Rhee (1996).

Figure 10. The $\left(\frac{V_{\text {bar }}}{V_{\text {cir }}}\right)_{R_{2,2}}$ versus $V_{200}$ for the DLO sample. Symbols are as in Fig. 6. DLOs belonging to haloes that include several objects (either DLOs, spheroids or irregulars) have not been considered. The shaded area shows the 95 per cent confidence interval for maximum discs (see text).

Figure 11. The $r_{\text {cross }}$ parameter divided by the virial radius $r_{200}$ versus the $V_{2.2}$ velocities for the DLO sample. Symbols are as in Fig. 6. DLOs belonging to haloes that include several objects (either DLOs, spheroids or irregulars) have not been considered.

Figure 12. The logarithmic slope, $L S$, versus the disc scale lengths $R_{\mathrm{d}}$ (a) and the $V_{2.2}$ velocities (b) for the DLO sample. Squares, triangles and circles correspond to DLOs formed in S1, S1b and S1c simulations, respectively. Open circles are data from spirals with extended H i RCs, in the same dynamical range (see table 2 in CvG91). 
Figure 13. The peak circular velocities $V_{\text {cir }}^{\text {peak }}$ versus the $R_{\text {peak }} / R_{\mathrm{d}}$ ratios for the DLO sample. Squares, triangles and circles correspond to DLOs formed in S1, S1b and S1c simulations, respectively. Dots are data from the Courteau sample of RCs in $\mathrm{H} \alpha$; open circles are data from spirals with extended H i RCs from table 4.1 in Rhee (1996). See text. 


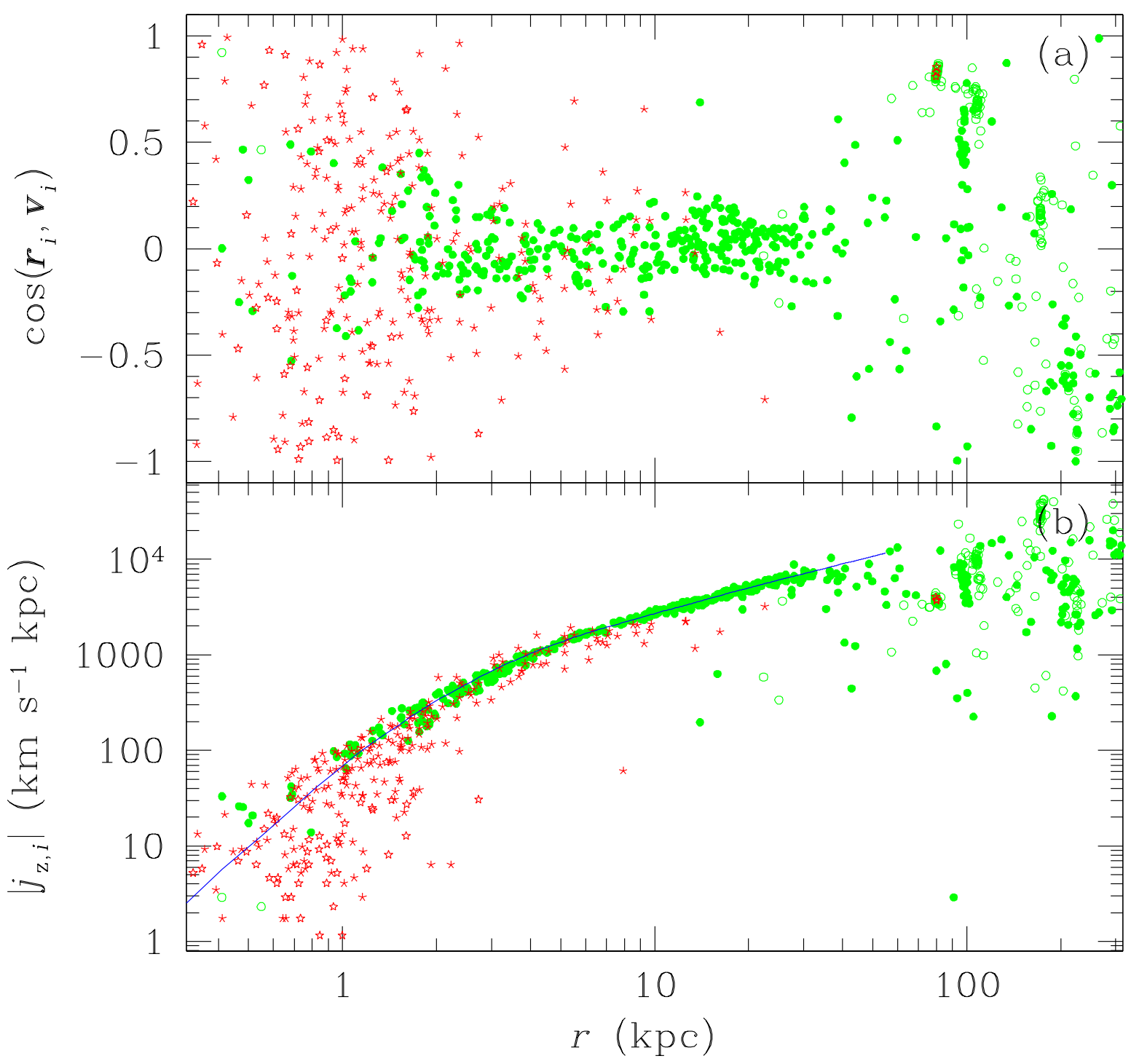

Fig. 1 


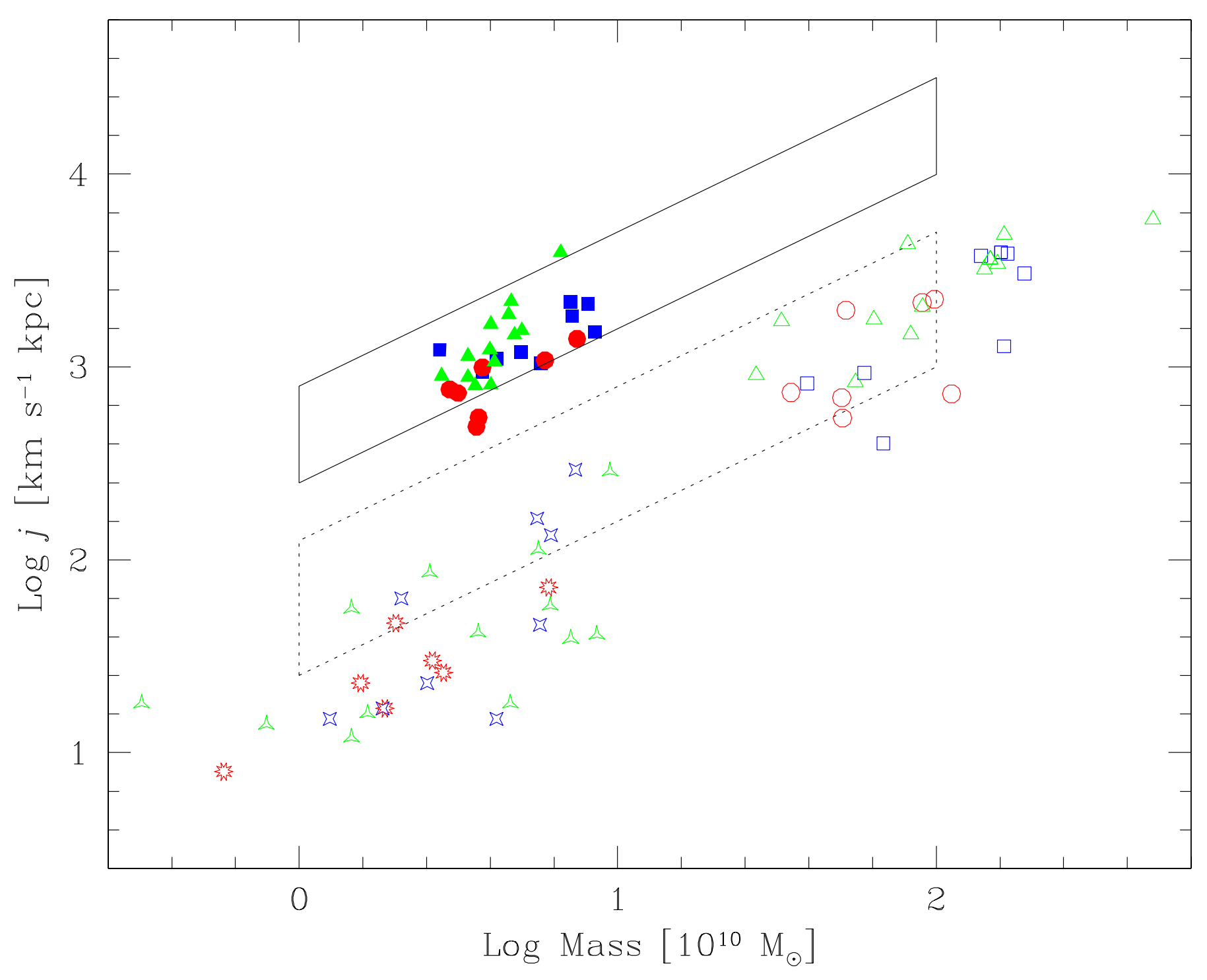

Fig. 2 


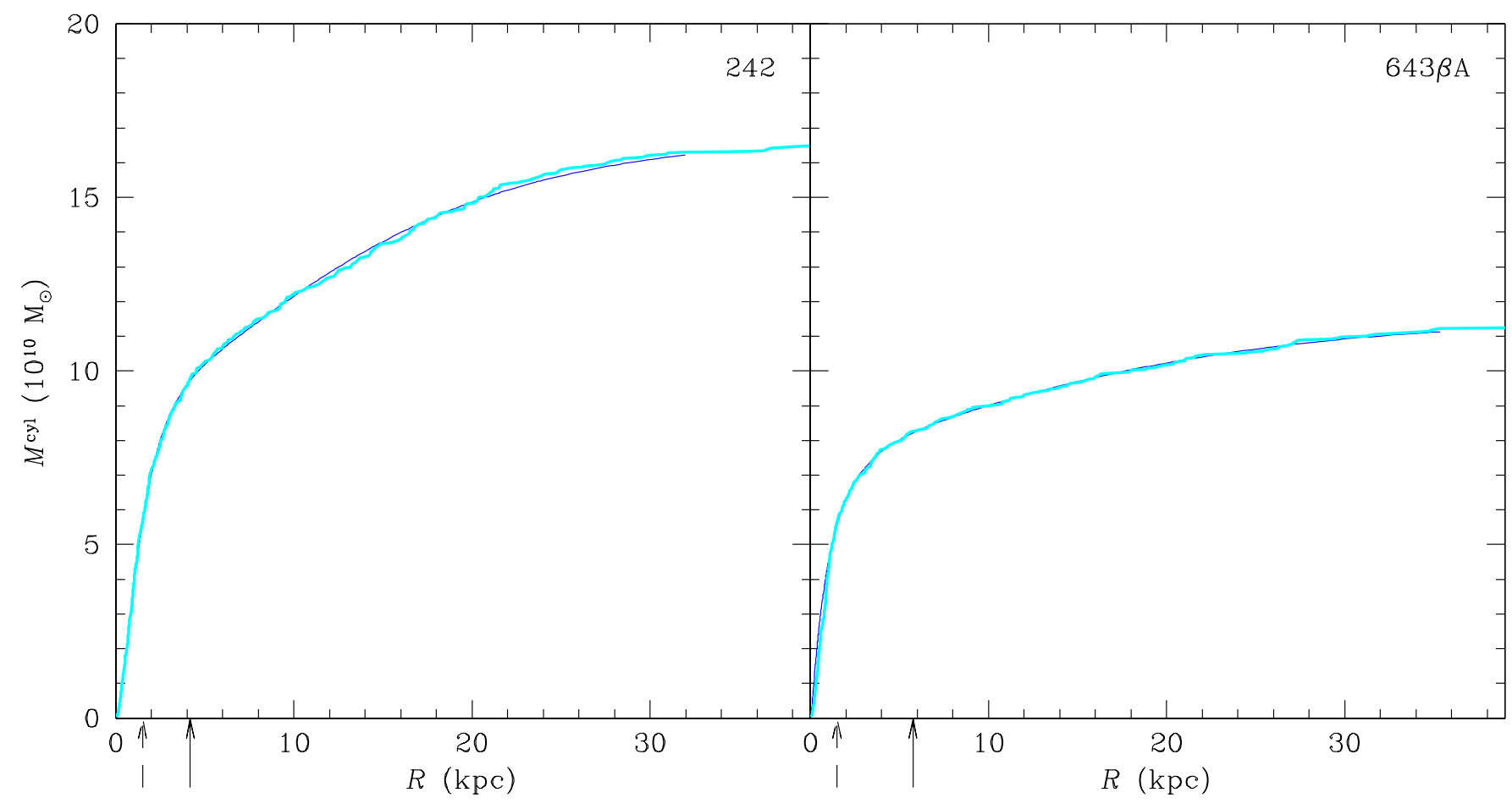

Fig. 3 


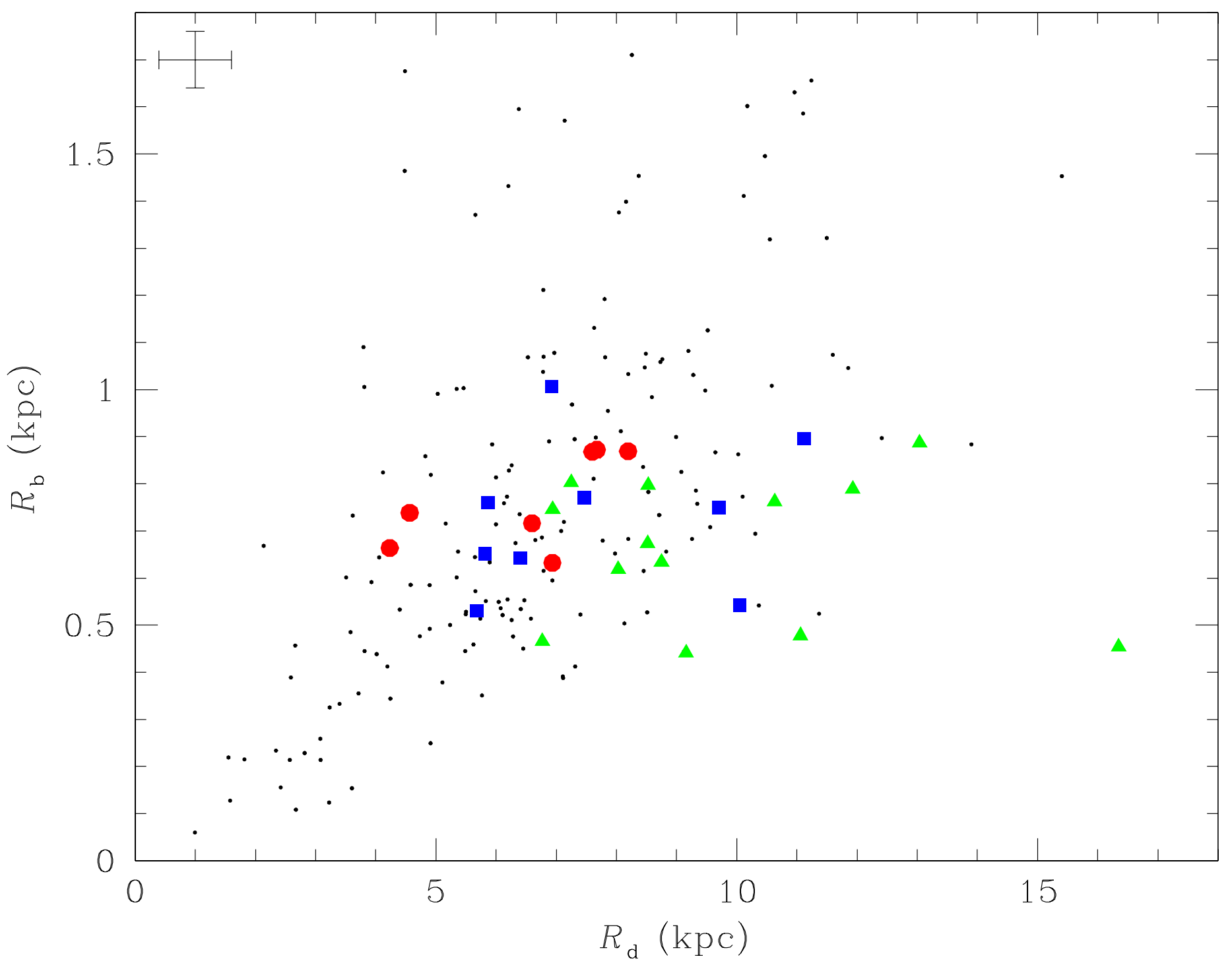

Fig. 4 


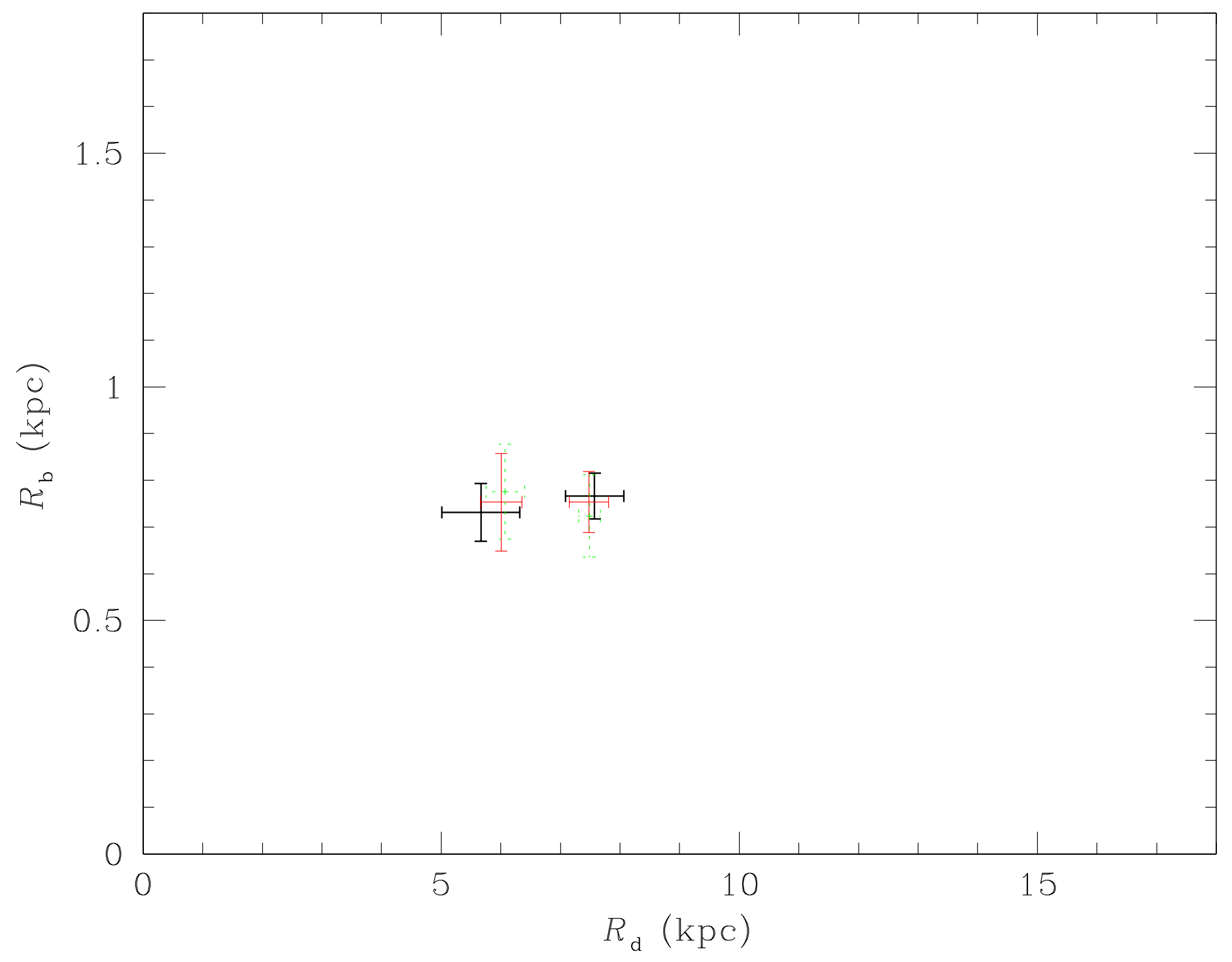

Fig. 5 


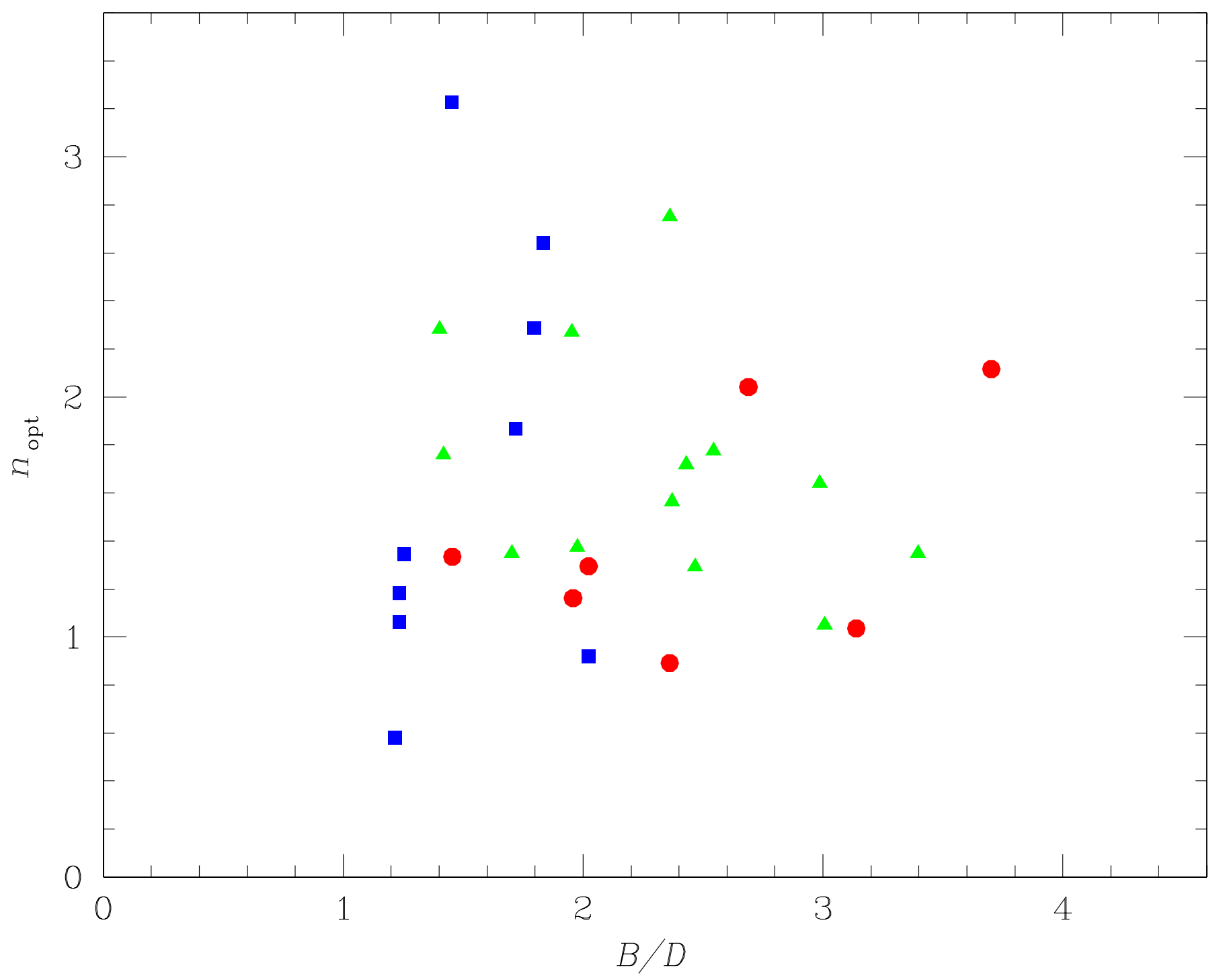

Fig. 6 


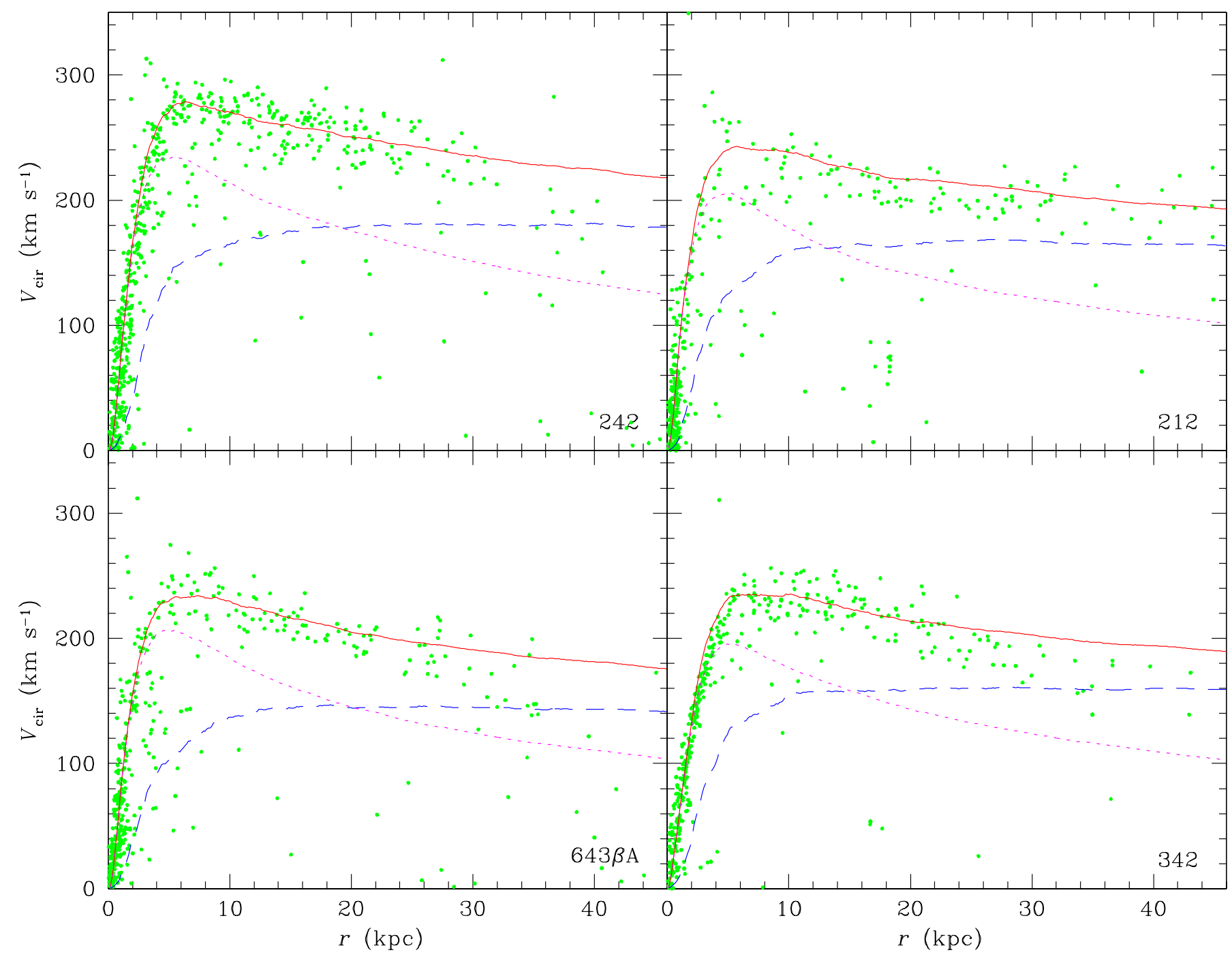

Fig. 7 


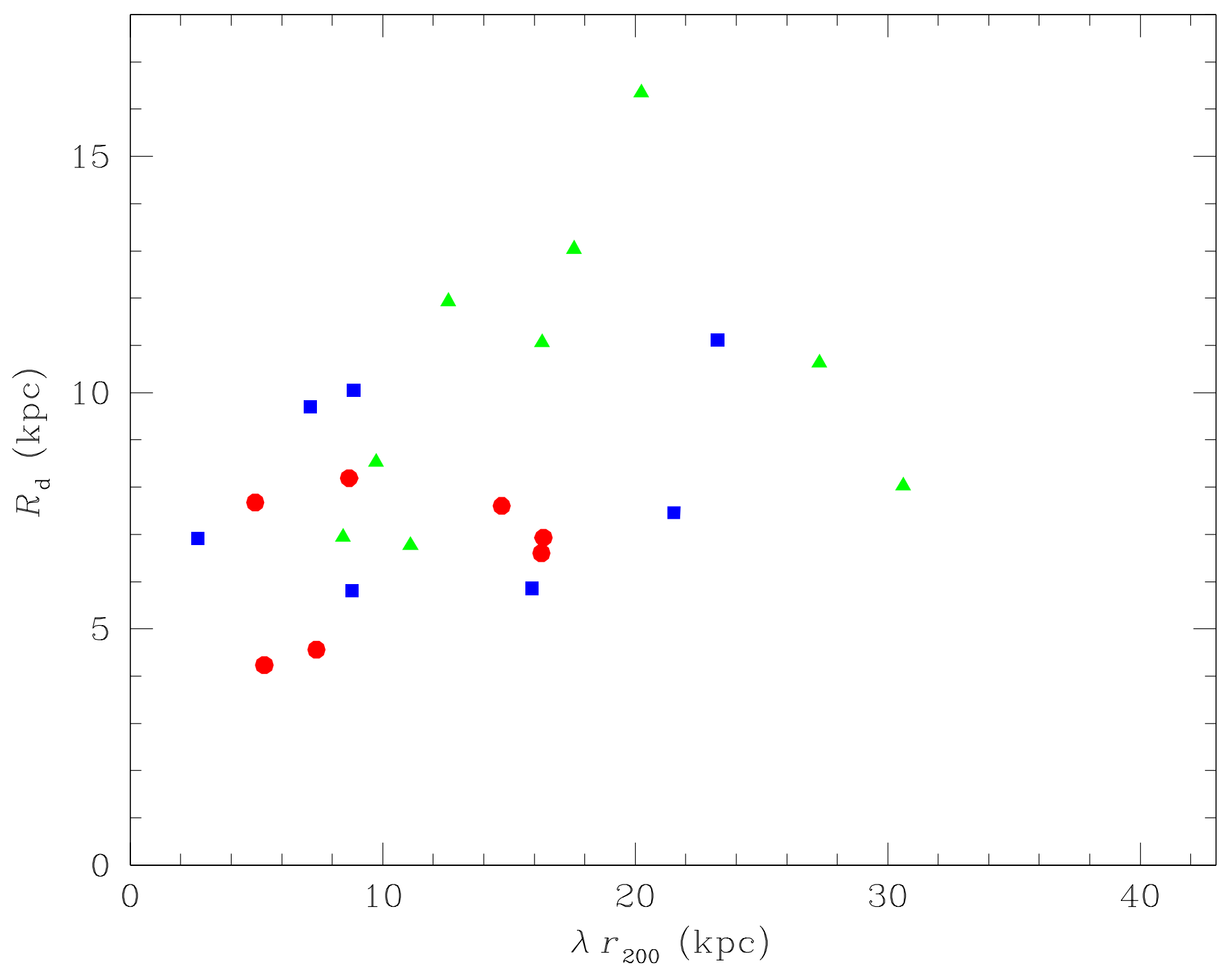

Fig. 8 


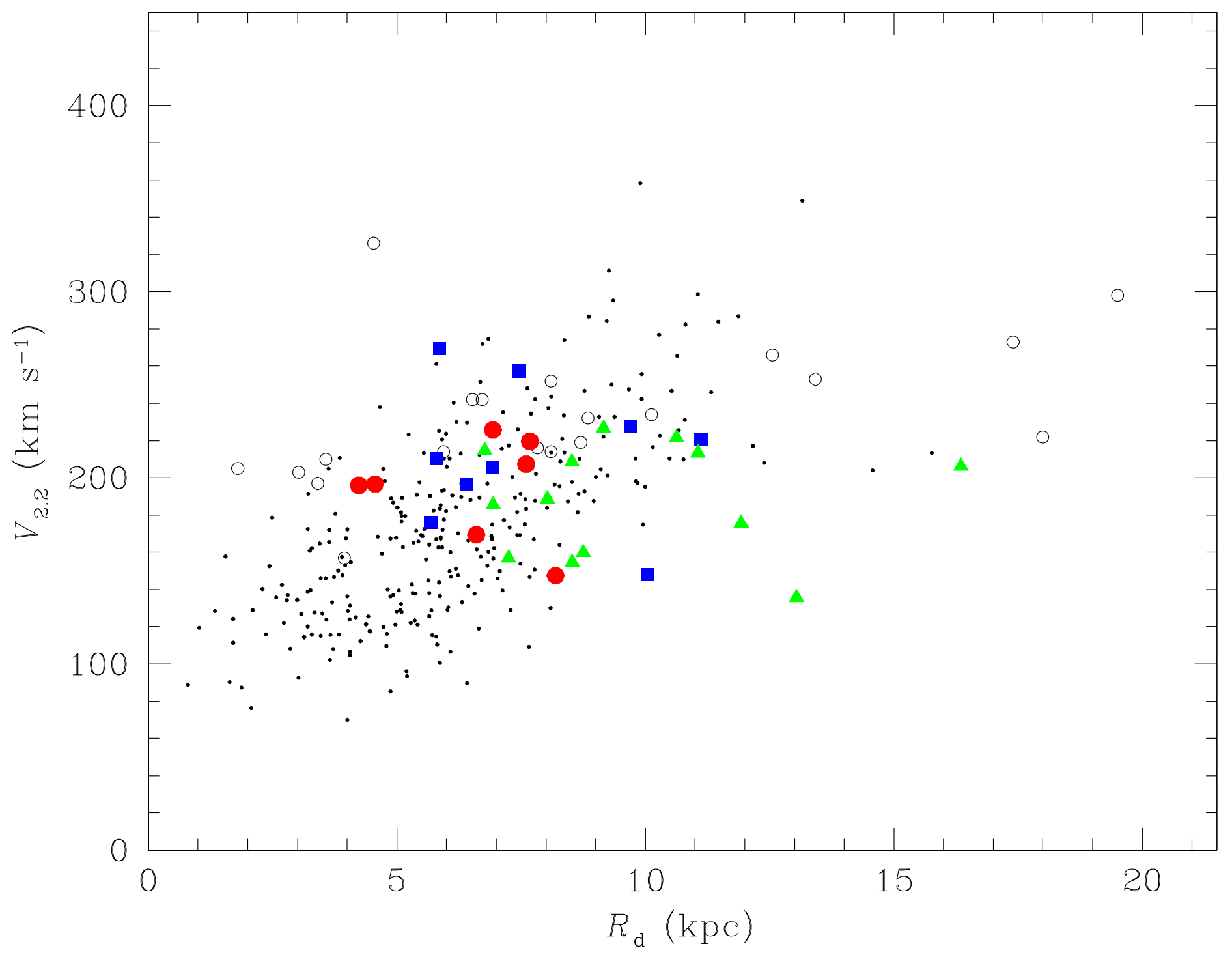

Fig. 9 


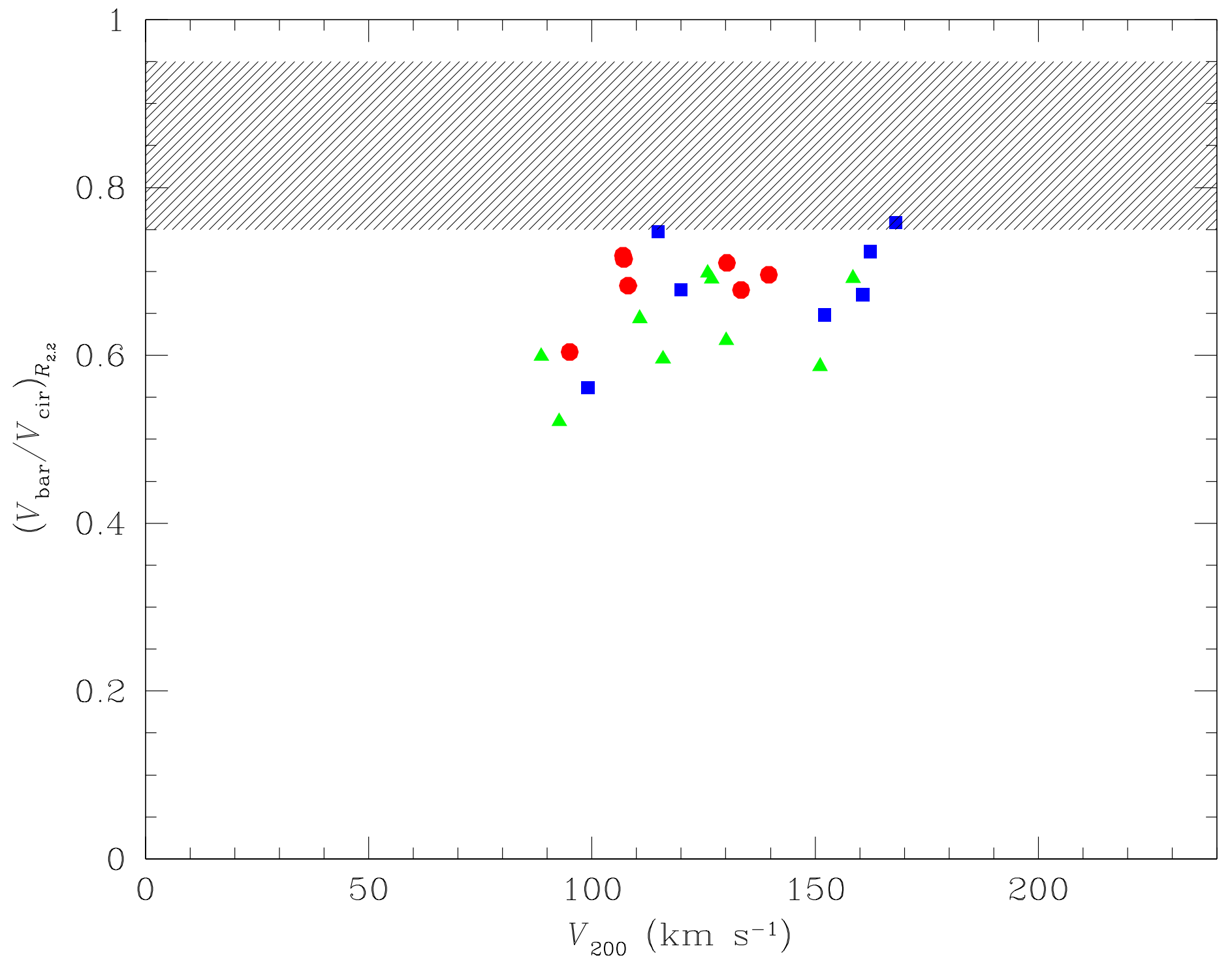

Fig. 10 


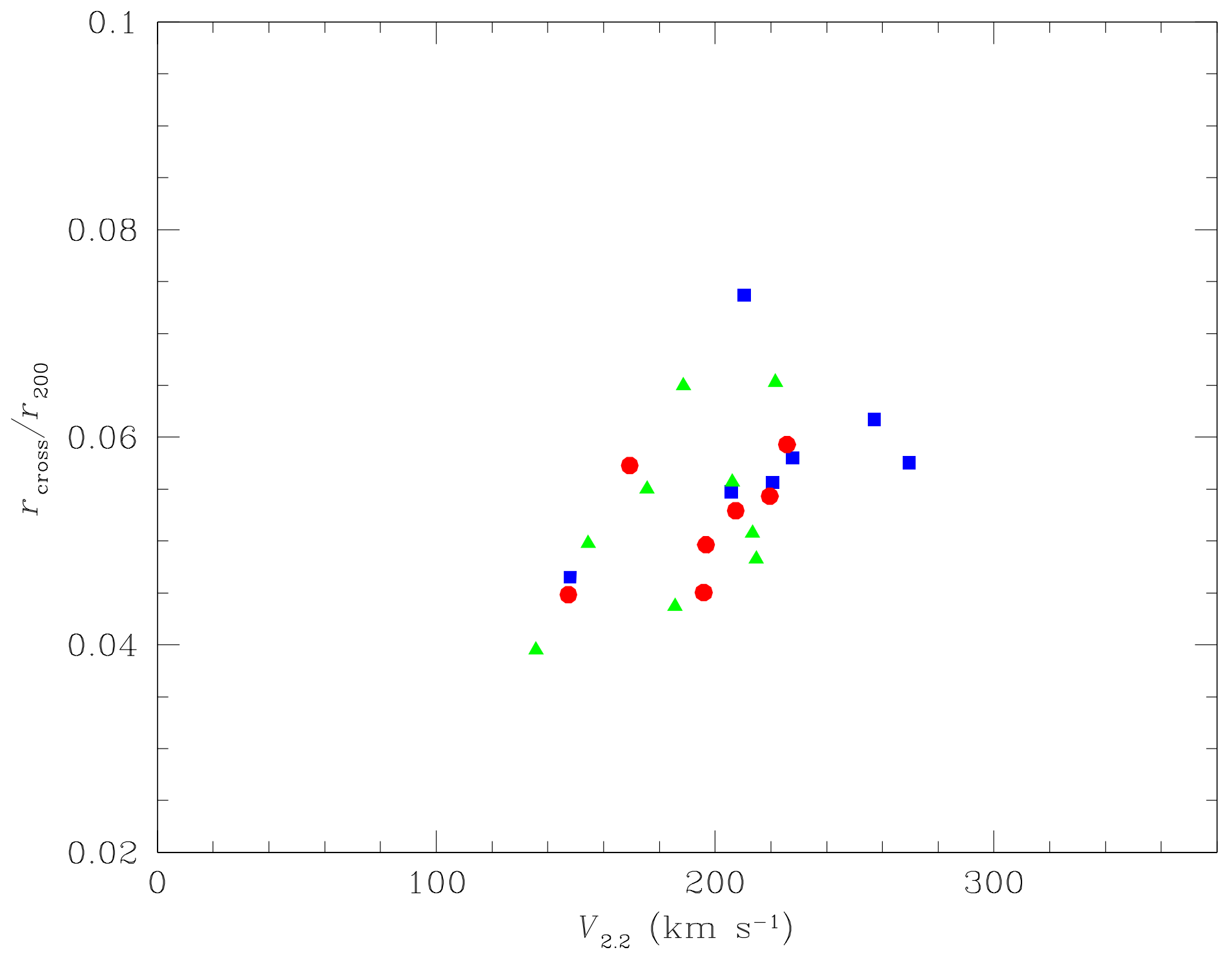

Fig. 11 


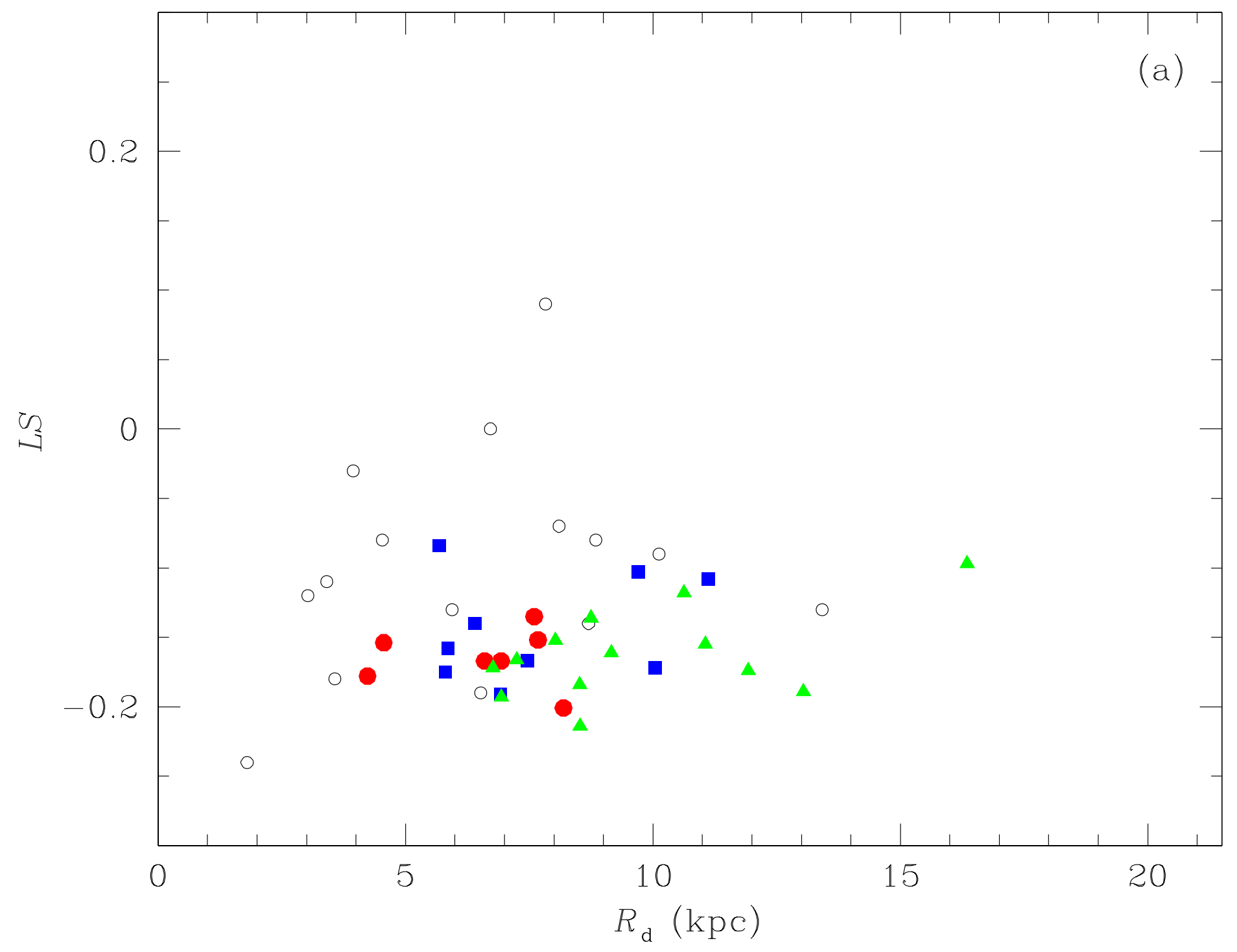

Fig. 12(a) 


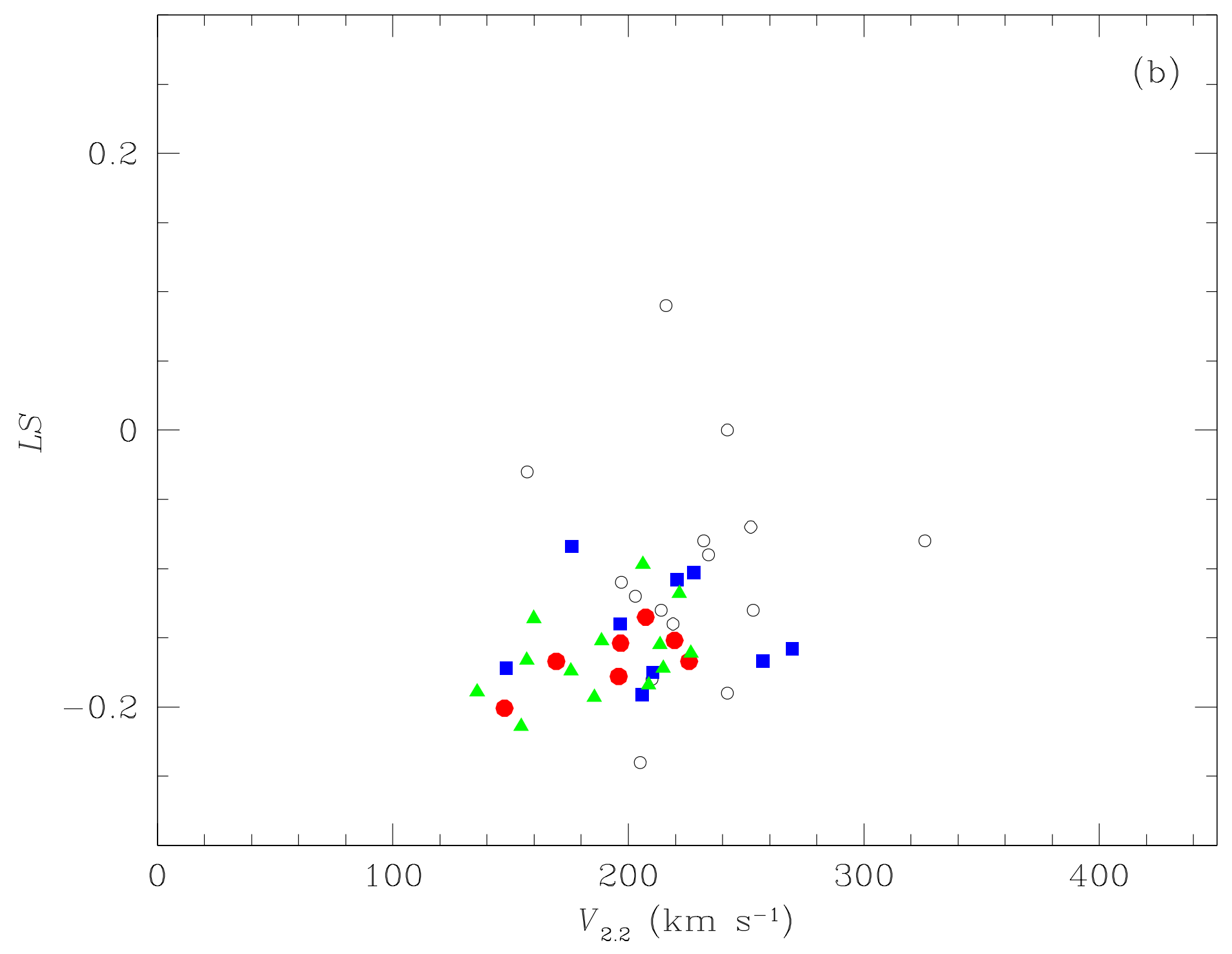

Fig. 12(b) 


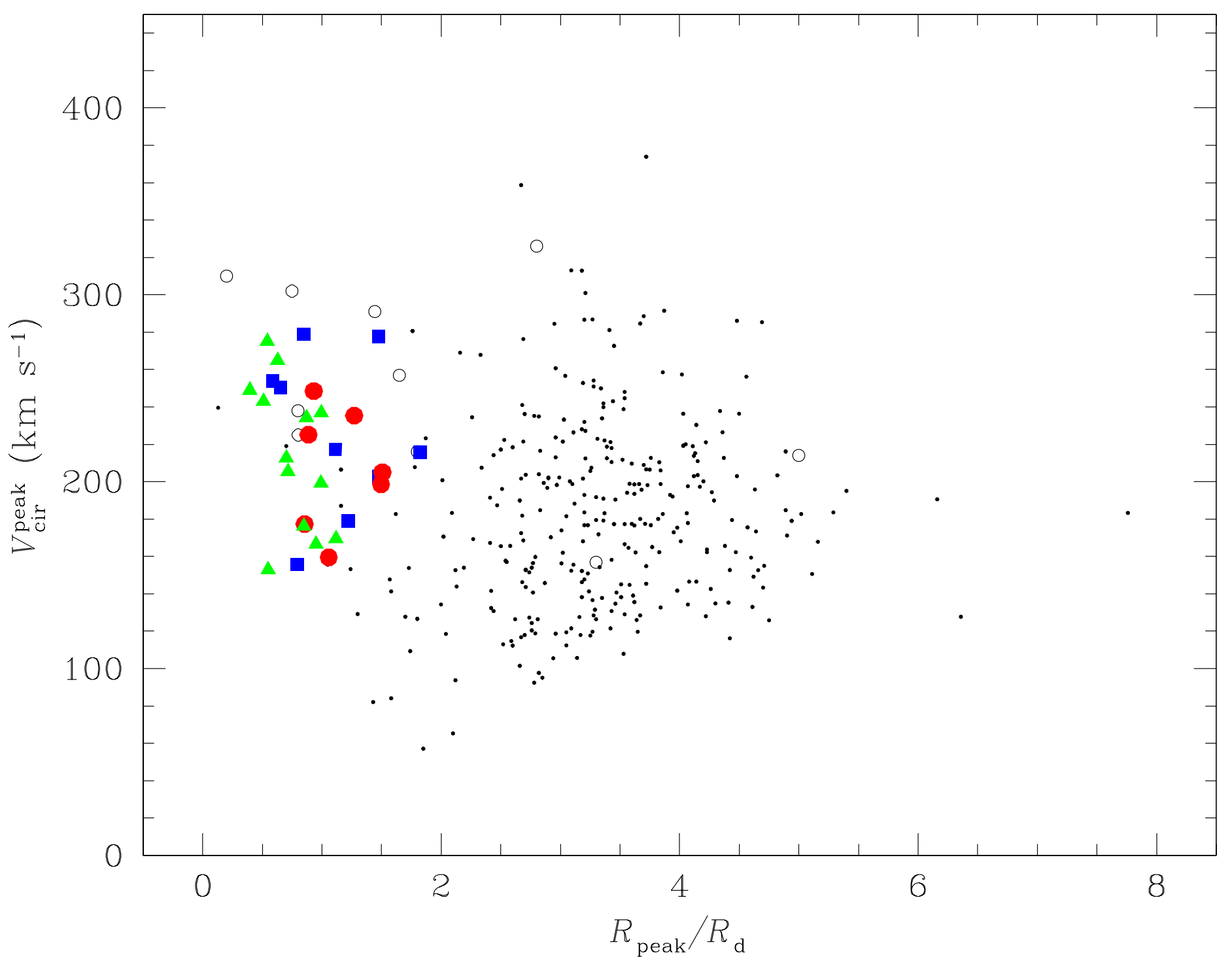

Fig. 13 